Check for updates

Cite this: J. Mater. Chem. B, 2021 9, 3640

Received 6th January 2021

Accepted 23rd February 2021

DOI: $10.1039 / \mathrm{d} 1 \mathrm{tb} 00033 \mathrm{k}$

rsc.li/materials-b

\section{Low-dimensional nanomaterials for antibacterial applications}

\author{
$X i-L e ~ H u,(D) \dagger^{a}$ Ying Shang, $\dagger^{a}$ Kai-Cheng Yan, D $\dagger^{b}$ Adam C. Sedgwick, (D) ${ }^{c}$ \\ Hui-Qi Gan, ${ }^{a}$ Guo-Rong Chen, ${ }^{a}$ Xiao-Peng He, ID *a Tony D. James (D) *be and \\ Daijie Chen (iD *d
}

\begin{abstract}
The excessive use of antibiotics has led to a rise in drug-resistant bacteria. These "superbugs" are continuously emerging and becoming increasingly harder to treat. As a result, new and effective treatment protocols that have minimal risks of generating drug-resistant bacteria are urgently required. Advanced nanomaterials are particularly promising due to their drug loading/releasing capabilities combined with their potential photodynamic/photothermal therapeutic properties. In this review, 0-dimensional, 1-dimensional, 2-dimensional, and 3-dimensional nanomaterial-based systems are comprehensively discussed for bacterial-based diagnostic and treatment applications. Since the use of these platforms as antibacterials is relatively new, this review will provide appropriate insight into their construction and applications. As such, we hope this review will inspire researchers to explore antibacterial-based nanomaterials with the aim of developing systems for clinical applications.
\end{abstract}

\section{Introduction}

The development of antibiotics in the 20th century resulted in the successful treatment of bacterial infections, which improved the quality of life of millions of patients worldwide. ${ }^{1-8}$ Unfortunately, their overuse and misuse has led to a rise in drug-resistant bacteria, which has rendered numerous antibiotics ineffective. This continuing trend poses a serious threat to the antibiotic development pipeline, which could eventually result in an inability to treat bacterial infections. $^{9-14}$ If not appropriately addressed, there are significant concerns that we will return to a "pre-antibiotic" era. ${ }^{15,16}$ Therefore, new and effective treatment strategies are urgently required, which will result in a reduced risk of developing drug-resistant bacteria.

\footnotetext{
${ }^{a}$ Key Laboratory for Advanced Materials and Joint International Research Laboratory of Precision Chemistry and Molecular Engineering, Feringa Nobel Prize Scientist Joint Research Center, School of Chemistry and Molecular Engineering, Frontiers Center for Materiobiology and Dynamic Chemistry, East China University of Science and Technology, 130 Meilong Rd, Shanghai 200237, China. E-mail: xphe@ecust.edu.cn

${ }^{b}$ Department of Chemistry, University of Bath, Claverton Down, Bath, BA2 7AY, UK. E-mail:t.d.james@bath.ac.uk

${ }^{c}$ Department of Chemistry, The University of Texas at Austin, Austin Texas 78712-1224, USA

${ }^{d}$ School of Pharmacy, Shanghai Jiao Tong University, 800 Dongchuan RD, Shanghai 200240, China. E-mail: cdj@sjtu.edu.cn

${ }^{e}$ School of Chemistry and Chemical Engineering, Henan Normal University, Xinxiang 453007, China

$\dagger$ Equal contribution.
}

In the area of materials sciences, graphene and various other carbon-based nanomaterials have emerged as excellent components for conductor and battery-based applications. ${ }^{17-19}$ The significance of these findings has inspired the design of new and improved nanomaterials and the identification of valuable optical and thermal properties. ${ }^{20,21}$ These important characteristics have attracted considerable interest from biomedical researchers who wish to repurpose these nanomaterials for diagnostic and therapeutic applications. ${ }^{22-25}$ Nanomaterials have now been tailored towards a range of biology-based applications, including use as drug delivery systems (DDSs) in order to enhance the selectivity and efficacies of specific therapeutics. ${ }^{26-29}$ In addition, nanomaterials have exhibited the ability to perform photodynamic/photothermal therapy (upon light irradiation, PDT uses photosensitizers to convert environmental oxygen to reactive oxygen species (ROS), and PTT converts photoenergy to heat upon light activation, respectively) for disease treatment. ${ }^{30}$ Moreover, nanomaterials co-loaded with therapeutic agents have been shown to provide synergistic effects with PDT/PTT, which reduces the required therapeutic dose and minimalizes off-target toxicities, ${ }^{31}$ while theranostics (combined therapeutic and diagnostic) has been developed where imaging agents have been loaded onto nanomaterials to facilitate the ability to "sense and treat".

Nanomaterials are defined as materials with at least one length dimension being less than $100 \mathrm{~nm} .{ }^{32}$ Recently, several reviews summarizing the development of nanomaterials for antibacterial applications have been reported. Niu and coworkers reviewed the antibacterial properties of different 
metals and metal oxides including titanium derivatives, zinc oxide, nickel, copper and copper oxide, gold, palladium, selenium and iron. $^{33}$ In another review, Truong and co-workers summarized the antibacterial mechanisms of different metallic nanomaterials. ${ }^{34}$ One of the most common mechanisms involves physical interaction with the bacterial surface, which kills bacteria through membrane damage. Which, results in ion release that causes enzyme inhibition or nucleic acid degradation, and ROS production. The authors concluded that there is an emerging trend for developing stimulus-activated nanomaterials, which require an external triggering element for the materials to function, affording "activatable" antibacterial materials. The most extensively used triggering elements include light (PDT/PTT) and magnetic force. Focusing on a variety of mechanisms facilitating antibacterial function, Song and co-workers prepared a comprehensive review on polymeric, antibiotic-free materials. ${ }^{35}$ The diverse antibacterial mechanisms include suppression of bacterial metabolism, catalytic bacterial killing by nanozymes and drug-loading/releasing. Moreover, the real-world applications of the as-developed strategies were deliberated for wound dressings, medical implants and food packaging. However, to the best of our knowledge, a summary of antibacterial nanomaterials in terms of their dimensionality has not been published (Scheme 1).

Dimensionality is an important asset of nanomaterials, which can change the intrinsic properties of the materials. Low dimensional materials including 0-D (zero-dimensional), 1-D (one-dimensional), 2-D (two-dimensional) and 3-D (threedimensional) nanomaterials have attracted the interest of materials physicists and chemists owing to their exceptional mechanical, magnetic, electric and optical properties. Significantly, differences in dimensionality could lead to different biological activities for the low-dimensional materials. The low-dimensional materials and their antibacterial mechanisms covered in this review are described in Table 1.

The dimensional difference of the materials causes their antimicrobial mechanisms to differ. For example, 0-D nanomaterials can serve as both a metal ion-releasing therapeutic agent and carrier for antibiotic delivery. Due to their small size, 0 -D nanoparticles can also be incorporated into hydrogels or loaded onto flat materials for practical applications. ${ }^{36}$ A representative clinical application is the use of a silvernanoparticle-based hydrogel for treatment of bacterial

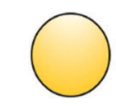

0-D Materials Metallic NPs Polymeric NPs $\ldots$

Antibacterial mechanisms: Oxidative stress, interruption of membrane integrity, biofilm inhibition, PDT/PTT induced cell death, interruption of protein synthesis, etc.

Scheme 1 Schematic illustration of the low-dimensional materials, including their antibacterial mechanisms, covered in this review. infection on the skin. ${ }^{33,34} 1$-D nanomaterials are upgraded in respect to surface area and are free from the drawbacks of internal deposition compared to 0-D, while they share properties with both 0 -D and their 2-D counterparts. 1-D rod-like nanomaterials are similar to 0-D with respect to performance and applications, while 1-D ribbon-like nanomaterials are more similar to 2-D because they are mostly derived from graphene. ${ }^{37}$ 2-D nanomaterials owing to their large surface area have been used for the loading of antibiotics and other therapeutic agents and combined with PDT/PTT. They can also be used for the direct eradication of bacteria by physical cutting as assisted by their sharp edge. ${ }^{37} 2$-D materials are also degradable in human bodies thus avoiding the toxicity brought about by 0-D or 1-D nanomaterials. ${ }^{38}$ 3-D nanomaterials are more likely to be a combination of 0-D, 1-D, or 2-D components, so they could exhibit the combined properties of 0-D, 1-D and 2-D nanomaterials resulting in composite systems with improved antibacterial properties. ${ }^{39}$ With the continuing development of materials science, the biocompatibilities of different nanomaterials have been greatly improved, which will stimulate the development of enhanced properties for improved clinical applicability. Within this review, to allow ease of understanding, we have separated antibacterial nanomaterials according to their dimensionality. In our review, we will discuss stimulus-activated and targetresponsive low-dimensional nanomaterials that have been recently developed for antibacterial applications, with particular emphasis on the parameters that determine their practical applicability including the antibacterial spectrum, biocompatibility for clinical use and selectivity towards other pathogens.

\section{0-D nanomaterials}

0-D nanomaterials are spherical-like materials with good dispersity and stability. These include metal-based nanoparticles, ${ }^{40,41}$ fullerenes, carbon dots, polymeric nanoparticles (polymer dots) and graphene quantum dots (GQDs). ${ }^{42,43} 0$-D nanomaterials are particularly attractive for antibacterial applications due to their high surface to volume ratios (drug loading/functionalisation) and ideal photophysical properties such as photoluminescence and PDT/ PTT. 0-D nanomaterials originated from research on metal nanoparticles, which could dissociate metal ions and exhibit an inherent antibacterial effect. Representative 0-D material-based antibacterial agents are silver nanoparticles (AgNPs), which have inherent therapeutic properties towards bacterial infection with possible mechanisms being not only metal dissolution but also electrostatic adsorption and photocatalysis. ${ }^{44,45}$ AgNPs are one of the first examples of a non-chemotherapy-based approach used in both fundamental and clinical research. ${ }^{46,47}$ In recent years, researchers have focused on enhancing the therapeutic potential of AgNPs. This includes the use of nanocrystalline cellulose (NCC) to generate AgNPs/NCC systems that display superior antibacterial activity when compared to AgNPs alone. ${ }^{48}$ Huang and co-workers synthesised stable AgNPs using $\gamma$-irradiation in the presence of a chitosan solution, which acted as a "green" stabilizing agent. This method generated AgNPs with significant antibacterial 
Table 1 Reported antibacterial mechanisms of low-dimensional materials covered in this review

\begin{tabular}{ll}
\hline & Composition of nanomaterials \\
\hline $\begin{array}{l}\text { 0-D nanomaterials } \\
\text { nanomaterials }\end{array}$ & $\begin{array}{l}\text { Nanocrystalline cellulose (NCC)-coated AgNPs } \\
\text { (AgNPs/NCC) } \\
\text { D-Cysteine-functionalised AgNPs (D-Cys-AgNPs) }\end{array}$ \\
& Dextrin-based nanocomposite hydrogels (Dex-G5-Ag) \\
& $\begin{array}{l}\text { Template-guided synthesis of ultrafine Ag NPs of } \\
\text { around 2 nm using water-soluble and biocompatible } \\
\gamma \text {-cyclodextrin metal-organic frameworks (CD-MOFs) } \\
\text { Integrating silver nanoparticles in situ into hydrogel } \\
\text { materials (AgNP-hydrogel) }\end{array}$ \\
$\begin{array}{l}\text { Mesoporous silica-supported Ag-bismuth (Bi) } \\
\text { Nanomaterials based on } \\
\text { other metallic and }\end{array}$ &
\end{tabular}

carbon-based particles
Antibacterial mechanism

AgNP-triggered oxidative stress, protein dysfunction, 48 and membrane and DNA damages

D-Cysteine inhibits biofilm formation, thus enhancing 50 the therapeutic effect of AgNPs

Controlled release of AgNPs, inducing oxidative stress, 57 protein dysfunction, and membrane and DNA damages $\mathrm{Ag}^{+}$ion release leading to protein dysfunction, and 54 membrane and DNA damages

Kinetically-controlled release of antibacterial silver ions 60

$\mathrm{Ag}-\mathrm{Bi} @ \mathrm{SiO}_{2}$ significantly increased local temperature 51 due to light absorption of BiNPs, leading to disruption of cell integrity and acceleration of silver ion release $\mathrm{CeO}_{2}$-decorated nanoparticle MOFs (MOF@CeO ${ }_{2} \mathrm{NPs}$ ) Inhibition of the function of extracellular ATP by $\mathrm{CeO}_{2} 70$ nanoparticles, leading to disruption of initial bacterial adhesion. In addition, planktonic bacteria are killed by cytotoxic reactive oxygen species (ROS) generated by the MOFs

Carbon nanodots (CNDs) (CND-250)

Nanomaterials based on Poly(ionic liquid)/PVA hydrogel

polymeric particles

Programmed cell death of bacteria induced by carbon 71 dots (C-dots) with different surface charges

Electrostatic interaction between phosphate groups in 58 the cell membrane of bacteria and the positive charges of the poly(ionic liquid) enhanced the cell membrane permeability, causing leakage of cytoplasmic contents. Dual PTT/PDT effect killing drug-resistant bacteria

nanoparticles (DMCPNs)

Organic nanoparticles based on polymers that have a Binding with bacterial LPS (lipopolysaccharides) to hydrophobic skeleton and hydrophilic side chain modified with protonated primary amines (PDCP) Porphyrin-based porous organic polymer, FePPOP ${ }_{\mathrm{BFPB}}$ disrupt membrane integrity

Catalytic transformation of $\mathrm{H}_{2} \mathrm{O}_{2}$ to ${ }^{\bullet} \mathrm{OH}$ species for bacterial eradication

Silver nanowires (AgNWs)

Co-V mixed metal oxide (MMO) nanowires $\left(\mathrm{Co}_{3} \mathrm{~V}_{2} \mathrm{O}_{8}\right)$

$\mathrm{Cu}^{2+}$-based coordination polymer nanofibers ( $\mathrm{CuO} / \mathrm{MnO}_{2}$ core nanostructures) PEG-functionalised AuNRs (PU-Au-PEG)

$\mathrm{Bi}_{2} \mathrm{~S}_{3}$-coated AuNRs (Au@Bi $\mathrm{S}_{3}$ ) $\gamma$-AlOOH, $\gamma$-MnOOH, and $\alpha-\mathrm{Mn}_{2} \mathrm{O}_{3}$ nanorods

Nanotubes

Nanoribbon-based supra-structure

2-D nanomaterials Graphene-based nanomaterials

$\mathrm{AgNO}_{3}$ mixed with $N, N$-bis(pyridyl-4-methyl)- $N$ fluorenyl-9-methoxycarbonyl (Fmoc)-L-glutamate (4MPFG) leading to gelation (4MPFG) Polycationic porphyrin $(\mathrm{Pp} 4 \mathrm{~N})$ mixed with GNR-PEO2000 (Pp4N/GNR)

GO and reduced GO (rGO)

GO destructs bacteria by cell membrane damage through chemical reactions, whereas rGO induces mechanical stress and pierces the cell membrane Cytoplasmic membrane damage facilitating reaction

Loading of sodium 1-naphthalenesulfonate (NA) onto reduced GO for chelation of AgNPs, producing AgNP-NA-rGO (AgNP-NA-rGO)

PEG-functionalised GO mixed with AgNPs (GO-PEG-Ag) Bacterial structure damage and production of ROS, causing cytoplasm leakage and metabolic disorder Membrane damage

Silver ion release by dissolution of the material after the 86 oxidation of metallic silver

Peroxidase-mediated transformation of low concentrations of $\mathrm{H}_{2} \mathrm{O}_{2}$ to toxic ROS species ROS generation and release of $\mathrm{Cu}^{2+}$ ions

Inhibition of bacterial adhesion and PTT for multidrug- 91 resistant bacteria

Dual-mode PTT/PDT

Firm bacterial adhesion causing cell wrapping and morphological disruption

Disruption of membrane integrity inducing DNA condensation

Dual-mode PDT/PTT

Loading of GO with AgNPs and $\mathrm{CoFe}_{2} \mathrm{O}_{4} \mathrm{NPs}$

$\left(\mathrm{Ag}_{-} \mathrm{CoFe}_{2} \mathrm{O}_{4}\right.$-GO)

GO nanocomposites assembled with QASs (quaternary Physical disruption of bacterial membrane ammonium salts)

2-D $\mathrm{MoS}_{2}$-based nanomaterials
Chitosan-coated 2-D $\mathrm{MoS}_{2}\left(\mathrm{CS} @ \mathrm{MoS}_{2}\right)$

AgBrNPs mixed with $\mathrm{MoS}_{2}$ nanosheets $\mathrm{AgBr} @ \mathrm{MoS}_{2}$ Membrane damage, and oxidation of intracellular proteins and lipids

PDT

Quaternized chitosan (QCS)-modified $\mathrm{MoS}_{2}$ nanoflakes Membrane adhesion followed by photothermal (QCS-MoS ${ }_{2}$ ) antibiotic release 
Table 1 (continued)

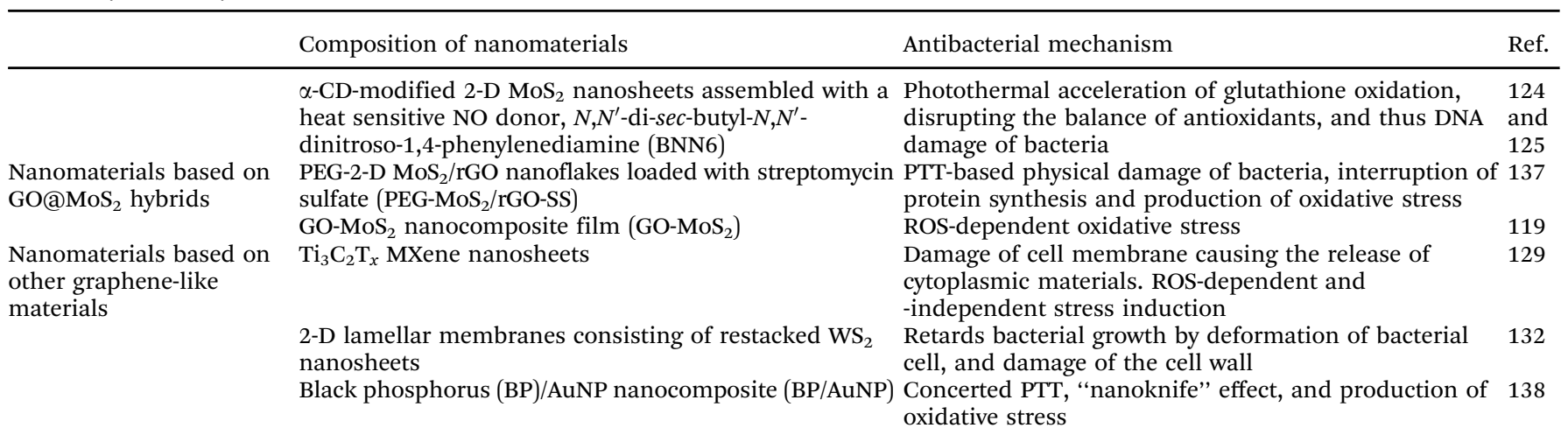

\section{3-D nanomaterials}

Metal-organic

frameworks
MOF@COF nanozyme

Mesoporous bioactive glass (MBG) loaded with in situ grown silver (Ag@pMBG)

$\mathrm{Ag}_{2} \mathrm{~S}$ nanoparticle- decorated nanocubes $\left(\mathrm{Ag}_{2} \mathrm{~S} / \mathrm{NCs}\right)$ Mg-Al double-layered rGO (Mg-Al@rGO)

AgNP-deposited carbon aerogels (p-BC/AgNP)

Defect-rich adhesive molybdenum disulfide $\mathrm{MoS}_{2} / \mathrm{rGO}$ vertical heterostructure (VHS $\mathrm{MoS}_{2} / \mathrm{rGO}$ )

Other 3-D nanomaterials Covalent-organic frameworks
$\mathrm{TiO}_{2}$ nanorod arrays

Degradable 3D-printed polylactic acid (PLA) scaffold
Tight capture of bacteria followed by in situ ROS generation

Silver ion release via molecular diffusion and degradation, leading to single-electron reduction of $\mathrm{O}_{2}$ interrupting the respiratory chain of bacteria

Dual-mode PTT/PDT

Protein degradation and GSH loss through the induction of oxidative stress

Silver ion release leading to protein dysfunction and DNA damage

Effective bacteria capture through local topological interactions, followed by ROS-based destruction of bacteria

Dual-mode PTT/PDT

Mitochondrial damage, leading to apoptosis
143

145

146

153

156

154

147 155 activity against Gram-positive and Gram-negative bacteria. ${ }^{49}$ Motivated to develop a more clinically relevant system, Ju and co-workers developed D-cysteine-functionalised AgNPs (D-Cys-AgNPs) for the enhanced treatment of planktonic bacterial biofilms (Gram-positive and Gram-negative). Biofilms are complex microbial communities encapsulated by extracellular polymeric substances (EPSs), which support bacterial growth and provide protection against antibacterial treatments. Using this strategy, D-Cysteine (D-Cys) inhibited biofilm formation, resulting in the dispersion of bacteria, which enhanced the therapeutic effect of the AgNPs. This approach was called the "disperse-then-kill" strategy. ${ }^{50}$ D-Cys-AgNPs displayed significant therapeutic efficacy towards $E$. coli, S. aureus, and P. aeruginosa, including their hard to treat biofilms. In addition, Ag-based 0-D nanomaterials have been used in combination with other systems to improve the overall therapeutic efficacy and to reduce side effects. One particular example includes the development of mesoporous silica supported Ag-bismuth (Bi) (Ag-Bi@ $\mathrm{SiO}_{2}$ ) nanoparticles by Dong and co-workers. ${ }^{51}$ The light irradiation (808 nm, $15 \mathrm{~min}$, and $1 \mathrm{~W} \mathrm{~cm}{ }^{-2}$ ) of $\mathrm{Ag}-\mathrm{Bi} @ \mathrm{SiO}_{2}$ resulted in a significant temperature increase (PTT) due to the light absorption of the BiNPs. This temperature increase accelerated the release of $\mathrm{Ag}^{+}$ions from the silica shell and provided an enhanced antibacterial effect. Using this approach, methicillin-resistant S. aureus (MRSA) and its biofilms were eradicated. Importantly, the clinical significance of $\mathrm{Ag}-\mathrm{Bi} @ \mathrm{SiO}_{2} \mathrm{NPs}$ was successfully demonstrated using mouse skin-infection models (Fig. 1). Other strategies include the combination of AgNPs with magnetic iron

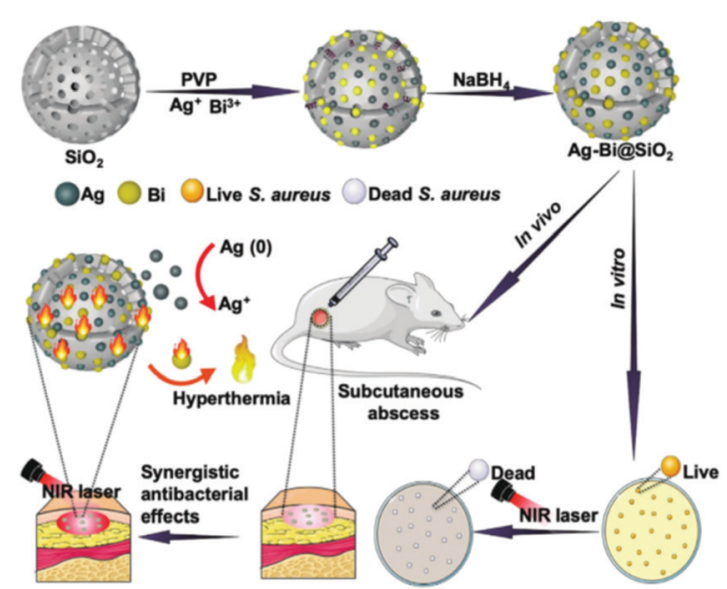

Fig. 1 Schematic illustration of $\mathrm{Ag}-\mathrm{BiaSiO}{ }_{2} \mathrm{NPs}$ that release $\mathrm{Ag}^{+}$ions and provide PTT for the treatment of bacteria in vitro and in vivo. Image reprinted with permission from ref. 51. Copyright 2020, Wiley-VCH.

oxide $\mathrm{Fe}_{3} \mathrm{O}_{4} / \mathrm{Fe}_{2} \mathrm{O}_{3} \cdot{ }^{52,53}$ Due to the challenges involved in synthesising and controlling the size and colloidal stability of AgNPs, Zhang and co-workers developed a template guided approach for the synthesis of ultrafine AgNPs using water soluble $\gamma$-cyclodextrin $\left(\gamma\right.$-CD) metal-organic frameworks (CD-MOFs). ${ }^{54}$ Peptide functionalisation (GRGDs (Gly-Arg-Gly-Asp-Ser)) of the MOFbased system facilitated platelet adhesion and aggregation at the injury site through peptide binding to the integrin GPIIbIIIa receptor. This reduced the time for blood to clot and 
promoted effective wound healing, where $\mathrm{Ag}^{+}$ion release resulted in the effective inhibition of $E$. coli and $S$. aureus.

Hydrogels are attractive for effective wound management, due to their excellent biocompatibility and therapeutic loading ability, including the incorporation of nanoparticles. ${ }^{55-58}$ More specifically, stimulus-responsive/smart hydrogels are particularly attractive for a range of biomedical applications; ${ }^{59}$ however, long term stability issues are often observed. To overcome this, Zhao and co-workers demonstrated that the simple loading of AgNPs into colour-responsive hydrogels prevented bacterial adhesion, hydrogel damage, and maintained the colour-responsive properties during application and long term storage. ${ }^{60}$ More recently, Dai and co-workers reported on the design of an acidresponsive hydrogel, Dex-G5-Ag, for the controlled release of AgNPs for the treatment of bacterial infection. ${ }^{57}$ Dex-G5-Ag hydrogels were formed by Schiff-base condensation between dextran (Dex-CHO) and an amino-functionalized dendrimer, G5-Ag (AgNPs encapsulated). The as-formed hydrogel was shown to degrade in an acidic environment and release AgNPs (Fig. 2). This strategy exhibited minimal cytotoxicity towards normal cell lines and proved effective for the treatment of a variety of bacteria including E. coli, P. aeruginosa, S. aureus, and S. epidermidis, and the effects were further evaluated using mouse-infection models. Other examples include the use of poly(ionic liquid)/poly(vinyl alcohol) (PVA) hydrogels for the development of antibacterial wound dressings. ${ }^{58}$

However, silver as a first-generation antibacterial material exhibits clear toxicity towards tissues, since it can be deposited in bodies leading to heavy-metal accumulation, which makes it hard for the approach to be clinically-accepted. Effective as it is, it could also kill other cells due to low selectivity. Functionalization or encapsulation of sliver could to some extent solve the problem but this approach is still far from satisfactory. Over the last several years, researchers have explored and evaluated the ability of non-silver-based nanomaterials for the treatment of bacteria. Firstly, other metals were evaluated, which mainly included a similar but much milder metal: gold. Gold nanoparticles (AuNPs)

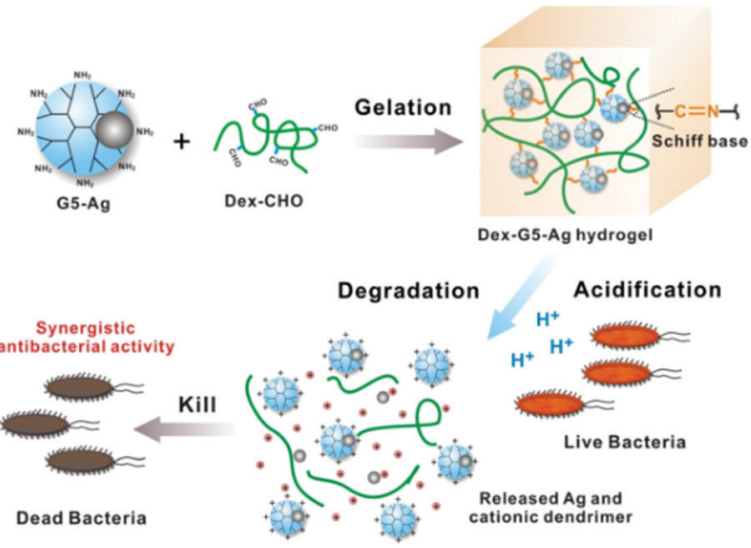

Fig. 2 Schematic illustration of the construction of an AgNP loaded hydrogel, Dex-G5-Ag, which displays potent antibacterial activity. Image reprinted with permission from ref. 57. Copyright 2018, American Chemical Society.

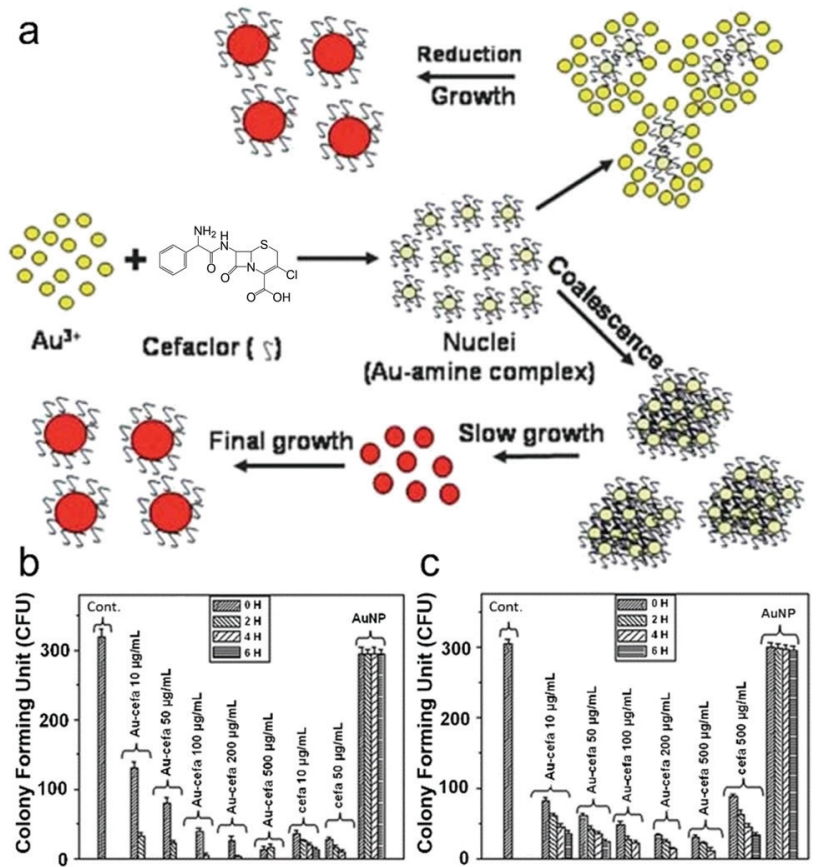

Fig. 3 (a) Schematic illustration of the synthesis of a AuNP-cefaclor complexed system for developing novel antimicrobial coatings. (b and c) Histogram plots showing antibacterial activity of the different systems on $S$. aureus (b) and E. coli (c) after different incubation times. Images reprinted with permission from ref. 65 . Copyright 2010, The Royal Society of Chemistry.

are well-established multifunctional 0-D nanomaterials used for biosensing, as drug carriers, as well as for theranostic, PDT/PTT and immunology applications. ${ }^{61-66}$ Perry and co-workers reported a unique antibacterial AuNP-strategy, in which gold ions $\left(\mathrm{AuCl}_{4}{ }^{-}\right)$ were reduced and capped by cefaclor a second-generation antibiotic (Fig. 3).$^{65}$ AuNP-cefaclor was shown to have potent antibacterial activity towards $S$. aureus and $E$. coli, while the individual use of AuNPs and cefaclor had minimal therapeutic effects (Fig. 3). Similarly, Jiang and co-workers explored AuNPs modified with 4,6-diamino-2-pyrimidinethiol (DAPT) as antibacterial agents (DGNPs). ${ }^{67,68}$ More recently, various particle sizes of DGNPs were evaluated against bacteria in order to correlate particle size with antibacterial activity. ${ }^{67}$ The antibacterial effects were assessed against Gram-negative and Gram-positive bacteria. Ultrasmall DGNPs $<2 \mathrm{~nm}$ (uDGNPs) displayed broad spectrum activity and as a result they were incorporated into agarose gels for wound dressing applications. The same authors evaluated a range of N-heterocyclic molecules with AuNPs, which displayed broad spectrum activity. Optimised antibacterial activities were achieved by varying the molar ratios of $\mathrm{N}$-heterocycles with the precursor of AuNPs, $\mathrm{HAuCl}_{4}{ }^{69}$ Compared to silver, gold-based agents are particularly delicate towards tissues, but this is also accompanied with a weakened antibacterial effect. Consequently, effective goldbased approaches are always assisted by using co-loaded antibiotics or other agents in order to attain acceptable performance.

Apart from the issues discussed above regarding AgNPs and AuNPs, these 0-D nanomaterials are expensive and result in metal ion release into the environment. These limitations 
prevent their scale-up and widespread use for clinical applications. Consequently, other metals are also being evaluated. Recently, Qu and co-workers developed $\mathrm{CeO}_{2}$-decorated nanoparticle $\mathrm{MOFs}$ (MOF@CeO ${ }_{2} \mathrm{NPs}$ ) as dual modal therapeutics for the treatment of planktonic bacteria and bacterial biofilms. ${ }^{70}$ Adenosine triphosphate (ATP) is an essential small molecule for all living organisms. More specifically, ATP plays a crucial role in bacterial adhesion and biofilm formation. Exploiting the known binding affinity of the lanthanide cerium (Ce) for ATP, $\mathrm{CeO}_{2}$ prevented biofilm formation through ATP extraction. Subsequent $638 \mathrm{~nm}$ light irradiation of the $\mathrm{MOF} @ \mathrm{CeO}_{2} \mathrm{NPs}$ facilitated the production of cytotoxic ROS to kill planktonic bacteria. The ROS generation was facilitated through a MOF consisting of zirconium ( $\mathrm{Zr}$ ) clusters and a PDT agent, tetracarboxyl phenylporphyrin. Overall, combination therapy using the MOF@CeO $\mathrm{NPs}_{2}$ was shown to be an effective treatment for bacteria and biofilm inhibition.

In order to further remove the toxicity of metals, other inorganic material systems were developed. A representative example was demonstrated by Yang and co-workers, in which carbon nanodots (CNDs) were designed to simultaneously treat and image bacteria. ${ }^{71}$ These antibacterial CNDs were developed using the wide-spectrum antibiotic metronidazole ${ }^{72}$ as the sole carbon source. CNDs-250 were prepared at $250{ }^{\circ} \mathrm{C}$ for $3 \mathrm{~h}$ and $8 \mathrm{~h}$, respectively (Fig. 4a). Evaluation of the optical density (OD) of bacterial solutions demonstrated a $71.7 \%$ inhibition of $P$. gingivalis at low concentrations $\left(1.25 \mu \mathrm{g} \mathrm{mL} \mathrm{L}^{-1}\right)$, similar to that of metronidazole (Fig. 4b). In contrast, when using control
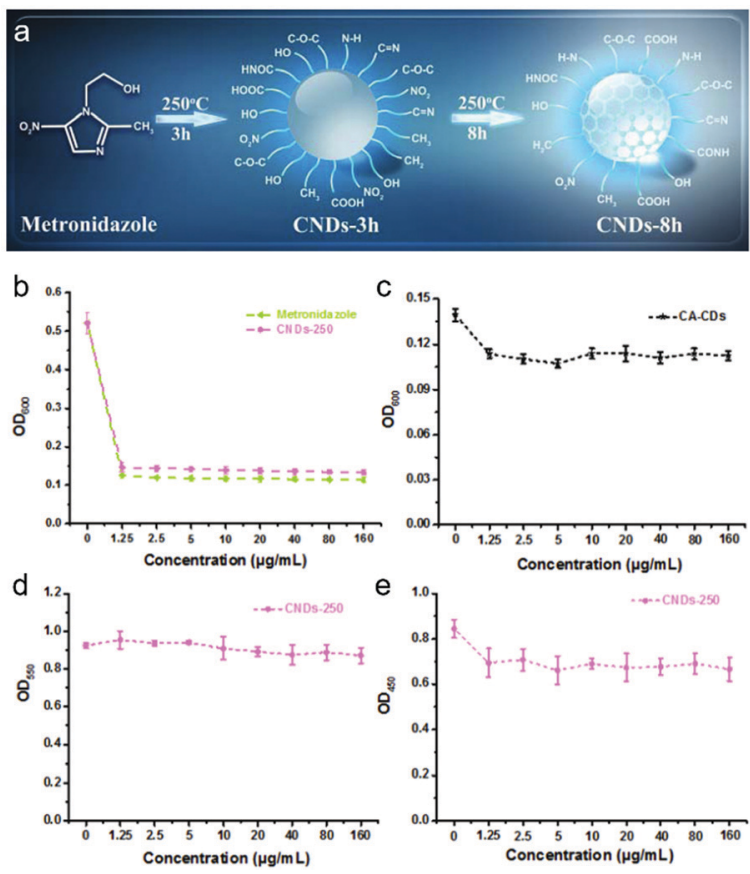

Fig. 4 (a) Schematic illustration of CNDs-3h and CNDs-8h prepared from metronidazole and differing by reaction time. (b and c) Growth curves of $P$. gingivalis after incubation with various concentrations of metronidazole and CNDs-250 (b), and CA-CDs (c) for 24 h. (d and e) Growth curves of S. mutans (d) and $E$. coli (e) after incubation with various concentrations of CNDs-250 for $24 \mathrm{~h}$. Images reprinted with permission from ref. 71 . Copyright 2017, The Royal Society of Chemistry. systems or treating other bacteria (Fig. 4c-e), the inhibition effects disappeared, thus demonstrating a selective effect towards $P$. gingivalis only. Based on this observation, the effects of CNDs-250 were further screened against a variety of bacteria, and it was found that CNDs-250 displayed significant activity towards obligate anaerobes (P. micros, $P$. intermedia, $P$. gingvivalis and Fusobacterium) rather than facultative anaerobes (S. mutans), obligate aerobes, or microaerophilic (E. coli) bacteria, which is similar to metronidazole which is the carbon source of the CNDs-250. The low toxicity towards human cell lines combined with their fluorescence properties enabled their use in cell labelling and imaging experiments. Importantly, CNDs were shown to be selective for bacteria in comparison to metronidazole only, displaying $60 \%$ cell viability in MC3T3-E1 cells. This underscores the significance of nanomaterials for antibacterial applications. MC3T3-E1 cells incubated with CNDs-250 displayed clear fluorescence emission when excited using green, blue, and UV light excitation, respectively. Another example reported by $\mathrm{Qu}$ and co-workers, ${ }^{73}$ evaluated the programmed cell death of bacteria induced by carbon dots (C-dots) with different surface charges. This report provides unique insights into the design of C-dots for antibacterial applications.

Yang and co-workers found that GQDs derived from graphene oxide (GO-GQDs) displayed weak antibacterial activity. This was believed to be due to their zero Gaussian curvature. In their study, they rationalised that the preparation of GQDs from $\mathrm{C}_{60}$ would provide a non-zero Gaussian curvature and provide improved antibacterial activity. These $\mathrm{C}_{60}$-GQDs exhibited significant antibacterial activity against $S$. aureus only (Fig. 5a), ${ }^{74}$ which was believed to be a result of the disruption of the bacterial cell envelope (confirmed by TEM images). Interestingly, E. coli was shown to have smooth surfaces regardless of the type of GQDs used (Fig. 5b). Scanning electron microscopy (SEM) images indicated that $\mathrm{C}_{60}$-GQD-treated $S$. aureus cells were coated with NPs, whereas, GO-GQD-treated $S$. aureus cells and all $E$. coli cells (no matter which type of GQDs was used to treat them) remained smooth (Fig. 5c). This selectivity for $S$. aureus demonstrated the importance of both the nanomaterial source and shape for physical contact-mediated treatment of bacterial species (i.e. S. aureus).

However, since all metallic and non-metallic elements are inevitably incompatible with tissues and can to some extent stay inside bodies for a long time, people have started to explore the use of polymers. Polymeric nanoparticles provide a low cost, highly degradable, and biocompatible alternative. Moreover, the homogeneity of polymeric nanoparticles is much better than metal nanoparticles, thus ensuring the stability of the antibacterial activity. A recent example was reported by Qin and co-workers, ${ }^{75}$ in which guanidine-based nanogels were developed that displayed antibacterial and antiadhesion properties. Significant antibacterial activity was observed against $S$. aureus and $E$. coli, which was successfully demonstrated using an infected mouse model. In addition, these nanogels were applied to the design of antimicrobial materials. A similar polymeric nanoparticle strategy was reported, which employed the antimicrobial functionality of $N$-halamine. 

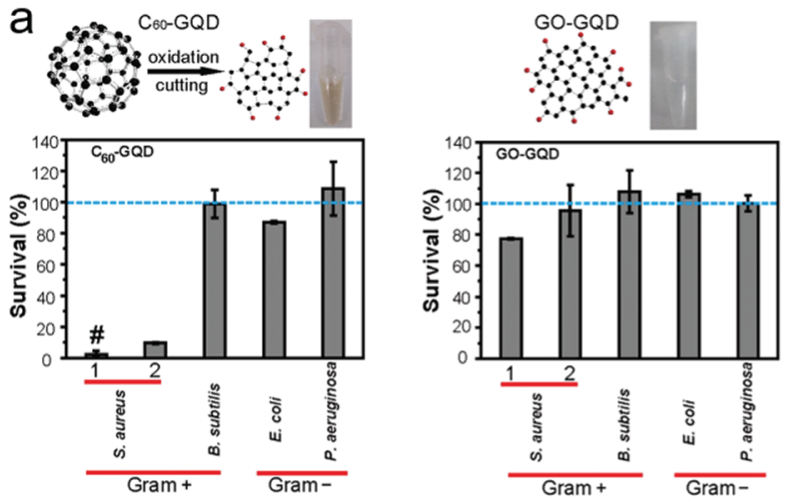

b

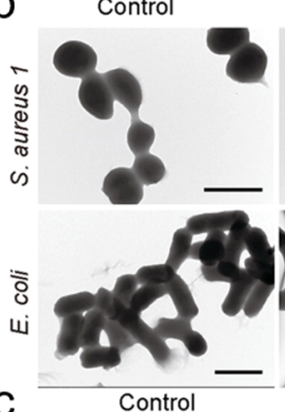

C60-GQD

GO-GQD
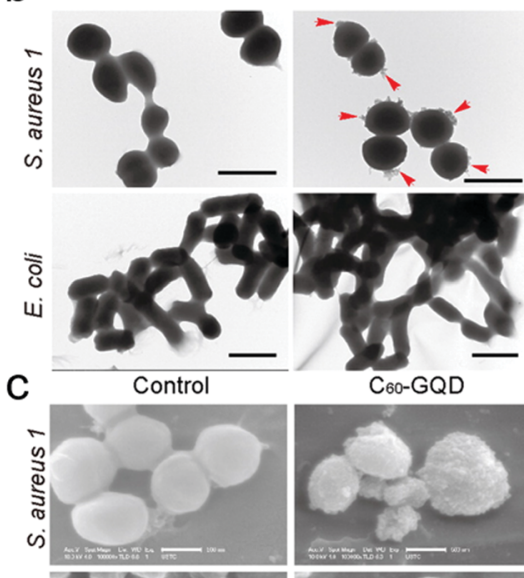
$\mathrm{C}_{60}-\mathrm{GQD}$
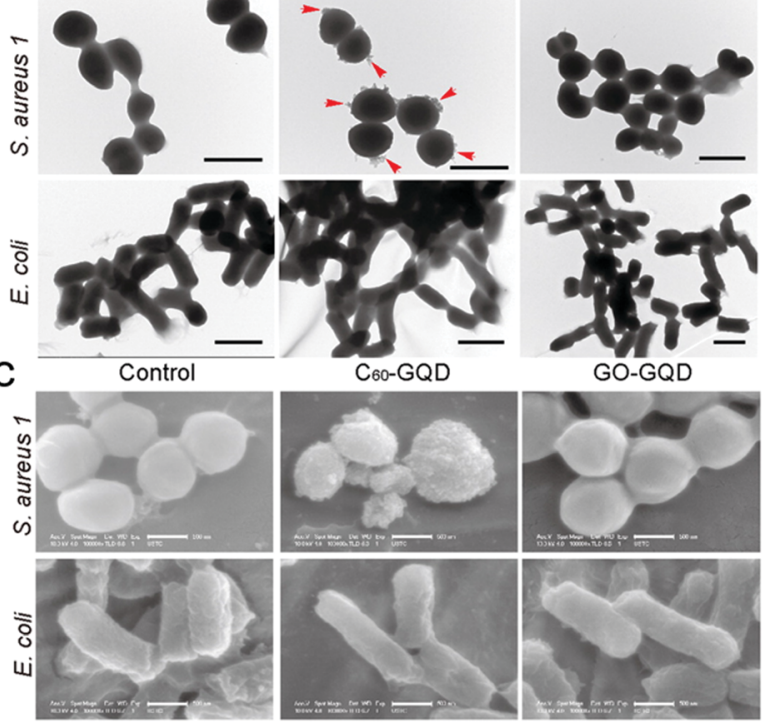

Fig. 5 (a) Bacterial assays using $\mathrm{C}_{60}-$ GQDs and GO-GQDs towards both Gram-positive and Gram-negative bacteria. (b and c) TEM (b) and SEM (c) images showing the surfaces of $S$. aureus and $E$. coli cells treated with $\mathrm{C}_{60^{-}}$ GQDs and with GO-GQDs along with control groups. Image reprinted with permission from ref. 74. Copyright 2015, American Chemical Society.

This system possessed strong antibacterial activity towards both $S$. aureus and E. coli and proved stable during long term storage. ${ }^{76}$ Zhao and co-workers reported biodegradable cationic $\varepsilon$-poly-L-lysine/poly(e-caprolactone) polymeric nanoparticles as new antibacterial agents towards B. subtilis, S. aureus, and E. coli. ${ }^{77}$ A photothermal agent poly(diketopyrrolopyrrolethienothiophene) (PDP/PTT) and photosensitizer poly[2methoxy-5-((2-ethylhexyl)oxy)-p-phenylenevinylene] (MEH-PPV) were dispersed in aqueous solution to form dual-mode conjugated nanoparticles, which displayed combined PDT and PTT effect upon activation by white light and $808 \mathrm{~nm}$ NIR light, respectively. ${ }^{78}$ The system was suitable for treatment of $E$. coli and exhibited no tissue toxicity. Very recently, new insights into polymeric nanoparticles have emerged. These include the synthesis of a monomer with two 1,2 3-triazoles and primary amines to improve water solubility and afford antibacterial activity, ${ }^{79}$ and a novel porphyrin-based porous organic polymer nanozyme prepared by the reaction of pyrrole and $\quad 4$-\{2,2-bis[(4-formylphenoxy)methyl]-3-(4-formylphenoxy) propoxy benzaldehyde (BFPB) that exhibited peroxidase-like activity toward a peroxidase substrate $3,3^{\prime}, 5,5^{\prime}$-tetramethylbenzidine (TMB) with $\mathrm{H}_{2} \mathrm{O}_{2}$ under $808 \mathrm{~nm}$ NIR irradiation. ${ }^{80}$ While Cheng and co-workers in 2021 developed a polymerantibiotic conjugate consisting of penicillin, 2-chloroethyl methacrylate (CEMA), and hydroxyethyl methacrylate (HEMA) crosslinked together and self-assembled into particles, as a strategy to develop resin-based restorative dental materials for sustained antibacterial therapy ${ }^{81}$ S. mutans biofilms resulting in dental caries were cultured and eradicated using the asdeveloped polymers, the slow antibiotic release discouraging bacterial resistance and ensuring long-term efficacy in the prevention of dental caries. Upon exposure to an enzymatic challenge resembling true intra-oral conditions, the efficiency of the antibacterial agent was maintained, demonstrating the potential for long-term effects and effective clinical behaviour.

\section{1-D nanomaterials}

1-D Nanomaterials are linear-based materials, which could have both stability/dispersity and capacity to load functional agents. Compared to 0-D, 1-D nanomaterials have a greatlyenhanced surface area and inherent photo-triggered therapeutic effects. These advantages have aroused significant interest and resultant development of these materials. Examples of such nanomaterials include nanotubes, nanowires, nanoribbons, nanofibers and nanorods, ${ }^{82}$ all of which exhibit exceptional thermal, electronic, mechanical, optical and magnetic properties. However, in comparison to 0-D nanomaterials, the development of 1-D nanomaterial technology was initially slow due to difficulties in synthesis and control of their morphology. ${ }^{82}$ 1-D nanomaterials are now fully established and used routinely in materials sciences and for biomedical applications. ${ }^{83,84}$ Firstly, ball-shaped metal particulates have been defined as 0-D, while rod-like materials are the archetypical 1-D nanomaterials, which initiated the 1-D materials area. Given that the main difference between these 0-D and 1-D metals is shape, metal-based 1-D rod-like metals are similar to 0-D metal nanoparticles in many aspects, and are attractive platforms for the development of effective antibacterial agents, ${ }^{85}$ which is a result of their inherent antibacterial activity (i.e. $\mathrm{Ag}$ and $\mathrm{Cu}$ ions) ${ }^{86}$ Recent reports include the electron beam (e-beam) irradiation of silver nanowire (AgNW) films. Surface irradiation (1200 kGy) altered the AgNW film surface morphology and chemical composition, which enhanced its antibacterial activity towards both E. coli and $S$. aureus. ${ }^{86}$ This example represents a simple method for enhancing the antibacterial properties of nanomaterials, however, the inherent toxicity of silver is still a big concern. In order to reduce toxicity, the approach is to control the release of silver. Liu and co-workers reported antibacterial $\mathrm{Ag}(\mathrm{I})$ metallogels formed by the selfassembly of nanotubes and nanofibers, confirmed by SEM and TEM images. The addition of an aqueous solution of $\mathrm{AgNO}_{3}$ to $N, N$-bis(pyridyl-4-methyl)- $N$-fluorenyl-9-methoxycarbonyl (Fmoc)-Lglutamate (4MPFG) resulted in instant gelation at room temperature. These metallogels displayed antibacterial activity 
against Gram-positive and Gram-negative bacteria and toxicity was reduced due to gel incorporation. ${ }^{87}$ Interestingly, this selfassembly was largely dependent on the gelator concentration, resulting in the formation of nanostructures ranging from nanotubes to nanofibers. Interestingly, the self-assembly of nanofibers was shown to result in increased damage to bacterial cell membranes when compared with nanotubes, which correlated with enhanced antibacterial activity. This research demonstrates the importance of nanomaterial morphology for efficient antibacterial applications.

With the aim of enhancing biocompatibility towards tissues, copper (Cu)-based and Au-based 1-D nanomaterials were proposed. Copper ( $\mathrm{Cu}$ )-based 1-D nanomaterials include $\mathrm{Cu} / \mathrm{C}$, $\mathrm{CuO}$ nanorods and nanoplatelets, which have exhibited comparable antibacterial efficacies to known antibiotics with enhanced biocompatibility. $^{88}$ More recently, Rauf and co-workers reported $\mathrm{Cu}^{2+}$-based coordination polymer nanofibers, ${ }^{89}$ that were formed from $\left[\mathrm{Cu}\left(\mathrm{H}_{2} \mathrm{O}\right)_{3}\right]^{2+}$ and HBTC $\left(1,3,5^{-}\right.$ benzenetricarboxylic acid) (Fig. 6a). The nanofibers exhibited excellent antibacterial effects against both $E$. coli and $S$. aureus (Fig. 6b). The proposed antibacterial mechanism was the generation of ROS combined with the release of $\mathrm{Cu}^{2+}$ ions. As with AuNPs, gold nanorods (AuNRs) have been shown to exhibit a range of diagnostic and therapeutic applications. ${ }^{90}$ A recent study by Zhao and co-workers demonstrated PEG-functionalised AuNRs for the PTT treatment of multidrug-resistant $S$. aureus and $P$. aeruginosa biofilms. ${ }^{91}$ These functionalised AuNRs were used with infected mouse models in vivo. Similarly, $\mathrm{Bi}_{2} \mathrm{~S}_{3}$-coated AuNRs ( $\left.\mathrm{Au} @ \mathrm{Bi}_{2} \mathrm{~S}_{3}\right)$ have

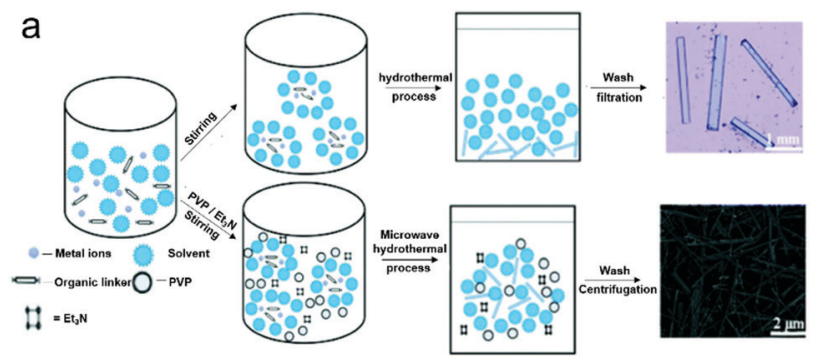

b

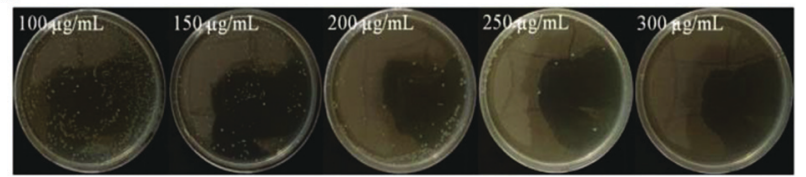

E. coli

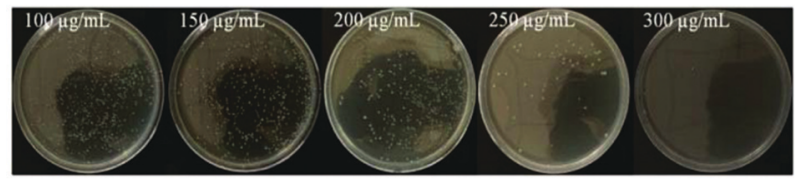

S. aureus

Fig. 6 (a) Basic schematic of the growth of the $\left[\mathrm{Cu}(\mathrm{HBTC})\left(\mathrm{H}_{2} \mathrm{O}\right)_{3}\right]$-based nanofibers. (b) Images of $E$. coli and $S$. aureus treated with different concentrations of $\left[\mathrm{Cu}(\mathrm{HBTC})\left(\mathrm{H}_{2} \mathrm{O}\right)_{3}\right]$-based nanofibers. Images reprinted with permission from ref. 89 . Copyright 2019, The Royal Society of Chemistry. been reported by Wang et al. for the light-based treatment of bacteria. $^{92}$ The NIR light irradiation (808 nm) of $\mathrm{Au} @ \mathrm{Bi}_{2} \mathrm{~S}_{3}$ resulted in the PDT/PTT-based treatment of E. coli and $S$. aureus (Fig. 7a), with clear inhibition seen in bacterial cultures (Fig. 7b). Interestingly, the individual use of each component resulted in minimal antibacterial activity being observed. As such, each component was required to have a good therapeutic effect.

In the area of 1-D research, oxides of metals have been investigated. For example $\mathrm{Mn}_{3} \mathrm{O}_{4}$-based nanorods and nanotubes have been developed by Chen et al., through a bi-directional-bidimensional growth model with biocompatibility comparable to gold systems. ${ }^{93}$ The antibacterial properties were confirmed using a range of bacteria including B. subtilis, S. aureus, S. faecalis, $P$. aeruginosa, and E. cloacae. The research further illustrated the importance of the morphology of nanomaterials, since $\mathrm{Mn}_{3} \mathrm{O}_{4}$ nanotubes were found to have a greater bacterial inhibition towards Gram-negative bacteria, than $\mathrm{Mn}_{3} \mathrm{O}_{4}$ nanorods. With a growing interest in 1-D nanomaterials as bacterial-resistant materials and for antibacterial applications, Selim and co-workers developed synthetic methods for the preparation of $\gamma$-AlOOH, $\gamma-\mathrm{MnOOH}, \quad$ and $\alpha-\mathrm{Mn}_{2} \mathrm{O}_{3}$ nanorods exhibiting biocompatibility/stability comparable to those of $\mathrm{Mn}_{3} \mathrm{O}_{4}$ nanorods. $^{94}$ The antibacterial activity was evaluated against P. aeruginosa, S. aureus, E. coli, B. subtilis, B. pertussis, and C. albicans, and all three nanorods exhibited significant antibiofilm activity, and $\alpha-\mathrm{Mn}_{2} \mathrm{O}_{3}$ was particularly effective against B. subtilis, $P$. aeruginosa, and B. pertussis biofilms. In particular the authors highlighted the importance of controlling the shape and the surface area of nanomaterials for achieving appropriate

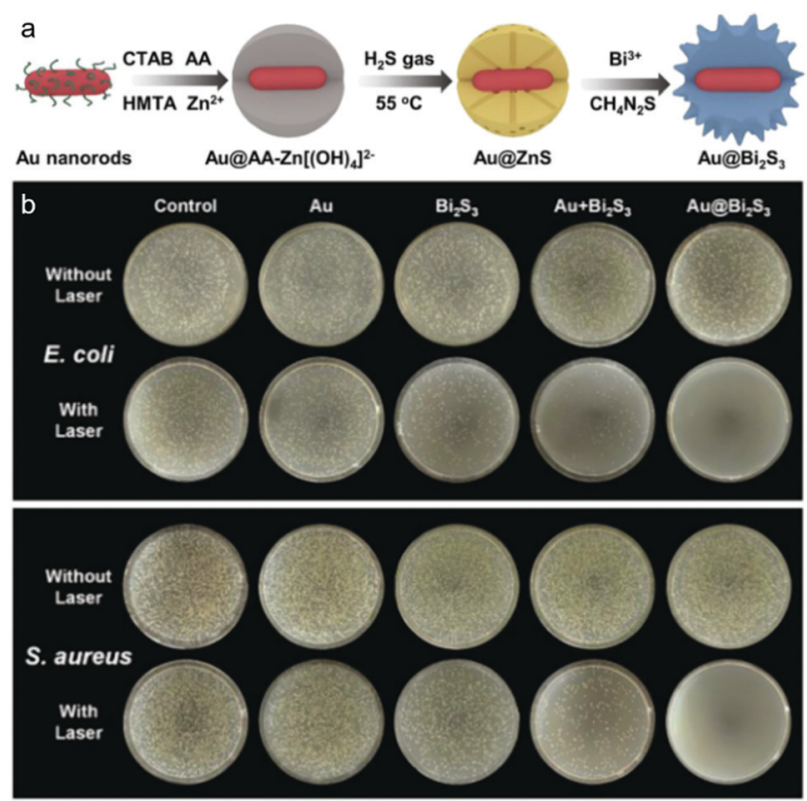

Fig. 7 (a) Construction of $\mathrm{Bi}_{2} \mathrm{~S}_{3}$-coated AuGNRs for the PDT/PTT-based treatment of bacteria. (b) Images of $E$. coli and $S$. aureus colonies on nutrition cultures after treatment with $A u \mathrm{ABi}_{2} \mathrm{~S}_{3}$ with and without laser irradiation. Images reprinted with permission from ref. 92. Copyright 2020, Elsevier B.V. 
antibacterial efficacy. It is important to note, that many researchers have endeavoured to improve the antibacterial efficacy of 1-D nanomaterials through functionalisation with antibacterial peptides. ${ }^{95}$ Wang and co-workers reported $\mathrm{Co}-\mathrm{V}$ mixed metal oxide (MMO) nanowires, which consisted of $\mathrm{Co}_{3} \mathrm{~V}_{2} \mathrm{O}_{8}$ dispersed amongst $\mathrm{Co}_{3} \mathrm{O}_{4} \cdot{ }^{96}$ Exploiting the intrinsic oxidase-like and peroxidase-like catalytic activities of Co-V MMO nanowires, in combination with low concentrations of $\mathrm{H}_{2} \mathrm{O}_{2}(50 \mu \mathrm{M})$, resulted in the successful treatment of $E$. coli. The antibacterial activity was attributed to the peroxidase-mediated transformation of $\mathrm{H}_{2} \mathrm{O}_{2}$ to the more harmful superoxide $\mathrm{O}_{2}{ }^{\bullet-}$. These materials offer an alternative to expensive $\mathrm{Ag}$ - and Au-based nanomaterials; however the homogeneity of suspension requires additional improvement. Importantly, the ability to use low concentrations of $\mathrm{H}_{2} \mathrm{O}_{2}$ minimises healthy tissue damage.

To solve the chronic problems caused by metals, 1-D researchers have developed systems from inorganic materials. The incorporation of non-metal/metal components into 1-D nanomaterials results in improved properties for sensing and therapeutic applications (e.g. electrochemical, fluorescence and PTT) ${ }^{97}$ Within this realm, graphene-derived 1-D nanomaterials have shown promise for antibacterial applications with not only PTT or agent-loading functions but also, a physical-cutting mechanism to assist therapy. ${ }^{98,99}$ For example, Davis and coworkers reported the combination of single-walled nanotubes (SWNTs) and lysozyme (displaying inherent antibacterial activity for Gram-positive bacteria) for the construction of multicomponent fibers. ${ }^{83}$ The cationic surfactant, tetradecyltrimethylammonium bromide was used to improve the stability of dispersions and enhance mechanical properties. CNTs are particularly attractive due to their tuneable thermal and electrical characteristics and high surface area to volume ratio. Additionally, CNTs have been shown as effective carriers for metallic NPs as well as increasing their aqueous stability and therapeutic properties. Hidal and co-workers reported the coating of multi-walled carbon nanotubes (MWCNTs) with AgNPs to develop an effective tool to prevent membrane fouling. In their study, the incorporation of AgNP-CNTs into polyethersulfone (PES) ultrafiltration (UF) membranes was efficient for antifouling applications with significant inhibitory activity towards both $E$. coli and $S$. Aureus being observed. ${ }^{100}$ With the AgNPs co-loaded into CNTs, the inherent toxicity was also avoided and the selectivity was improved. Hao et al. reported MWCNT-glucosamine-AgNP nanocomposites for the construction of functional materials with long-term antibacterial activity. Using this strategy, MWCNTs were grafted with glucosamine, which facilitated the coordination of AgNPs (Fig. 8a-d). ${ }^{101}$ This approach permitted the slow and controlled release of AgNPs, which provided long-term antibacterial activity against $S$. aureus over 35 days (Fig. 8e) with minimal side effects.

When compared with antibacterial drugs, the effects of 1-D nanomaterials that rely solely on photophysical mechanisms are insufficient. Within our group, graphene nano-ribbons (GNRs) have been evaluated for PDT/PTT applications. GNRs exhibit excellent mechanical strength and good biocompatibility and with co-loaded PDT agent can afford potent antibacterial

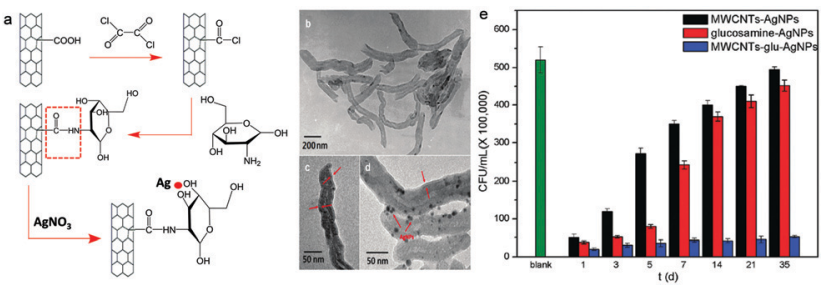

Fig. 8 (a) Basic schematic for the development of glucosamine-grafted CNTs and subsequent AgNP coordination. $(b-d)$ TEM images of the CNTs (b), CNTs with glucosamine (c), and CNTs with glucosamine and AgNPs (d). (e) S. aureus survival rates in Luria-Bertani medium with treatments of MWCNT-AgNPs (control), glucosamine-AgNPs (control), and MWCNTglucosamine-AgNPs during a 35 day period. Images reprinted with permission from ref. 101. Copyright 2017, The Royal Society of Chemistry and the Centre National de la Recherche Scientifique.

effects comparable to those of antibacterial drugs. ${ }^{102,103}$ Through our work, we have developed a series of structurally well-defined GNRs functionalised with hydrophilic flexible poly(ethylene oxide) (PEO) chains to reduce the toxicity and improve hydrophilicity. ${ }^{85}$ GNR-PEO exhibited aqueous dispersion/ stability suitable for biological applications. Evaluation of the morphology of these systems revealed hierarchical selfassembled GNR-PEO aggregates as supramolecular nanostrips, which subsequently formed ultralong nanobelts. In addition, the near-infrared (NIR) absorption (750-850 nm) of GNR-PEO permitted its use as PTT agents. PTT was demonstrated in combination with a porphyrin (PDT agent) as a dual wavelength $(660+808 \mathrm{~nm})$ PDT/PTT therapeutic. ${ }^{103}$ Specifically, polycationic porphyrin $(\mathrm{Pp} 4 \mathrm{~N})$ was mixed in aqueous solution with GNRPEO2000, which resulted in $\pi-\pi$ stacking interactions and formation of a Pp4N/GNR nanocomposite. The positively charged ammonium groups on $\mathrm{Pp} 4 \mathrm{~N}$ acted as targeting moieties for the negatively charged bacterial surfaces (Fig. 9a). Pp4N/GNR nanocomposite exhibited synergistic antibacterial effects under dual irradiation $(660 \mathrm{~nm}+808 \mathrm{~nm})$ and was successfully applied for the treatment of $A$. baumannii and methicillin-resistant $S$. aureus (MRSA) infected wounds in mouse models (Fig. 9b).

\section{2-D nanomaterials}

While 1-D nanomaterials can be used for numerous applications, many issues remain including limited surface area and loading capacity. Moreover, a lone PTT function could not generate significant clinical activity, which prevented them from becoming comprehensive therapeutics. Consequently, 2-D nanomaterials were developed as novel large-surface area and platform-like nanosystems with combined drug/PDT/PTT functionality. 2-D nanomaterials represent a class of nanomaterials that possess sheet/layered-like structures. Compared with 0-D and 1-D, these unique structural features afford unprecedented physical, electronic and chemical properties, which have led to their exploration in electronics/optoelectronics, electrocatalysis, batteries, supercapacitors, solar cells, photocatalysis, and sensing applications. ${ }^{104}$ Moreover, the sheet-like nature of 2-D platforms equips them with a large surface area, which is 

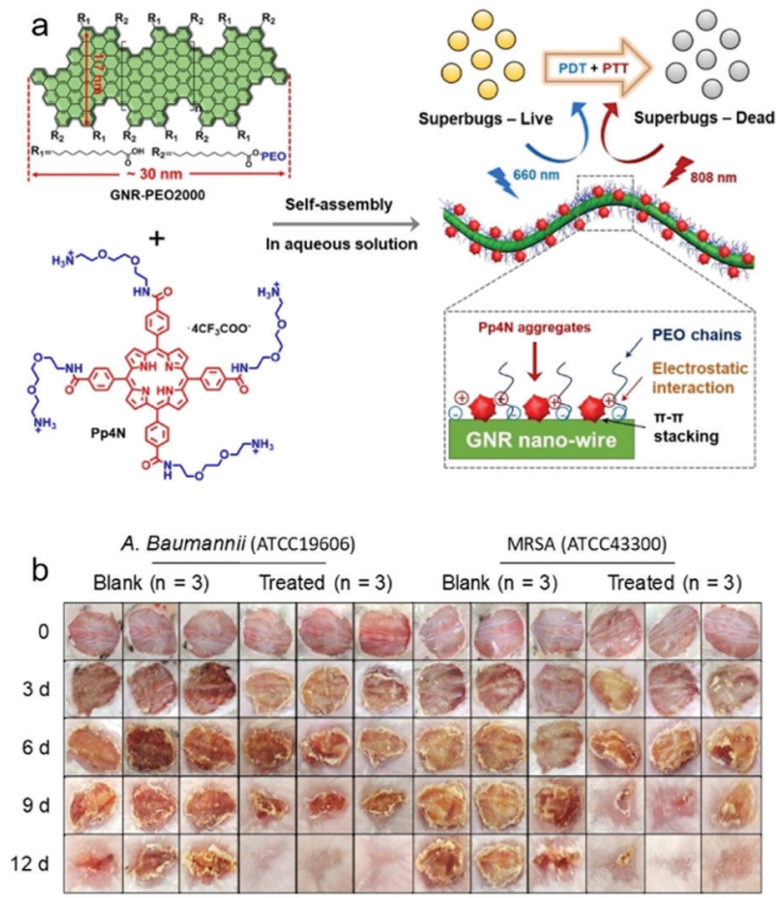

Fig. 9 (a) Basic schematic of the Pp4N/GNR nanocomposite strategy for the light-based treatment (PTT/PDT) of bacteria. (b) Photographs of A. baumannii- and methicillin-resistant S. aureus (MRSA) infected wounds with and without treatment over a 12-day period. Images reprinted with permission from ref. 103. Copyright 2019, Wiley-VCH.

highly photoreactive with improved physical contact and PDT/ PTT performance. As such these 2-D materials can be used to develop dressings, and can be co-loaded with 0-D, 1-D, drugs, or photosensitizing agents to provide synergistic effects. In general, due to the increased surface area, 2-D nanomaterials possess all the advantages of 1-D nanomaterials and in addition exhibit high agent-loading and are degradable/biocompatible when compared with 0-D nanomaterials. ${ }^{104}$ More specifically, a range of 2-D nanomaterials have been developed and explored for antibacterial applications. These include graphene-based, transition metal dichalcogenide, transition metal oxide and transition metal hydroxide 2-D nanomaterials. ${ }^{105,106}$ The large surface area of 2-D nanomaterials facilitates the incorporation of a range of therapeutics; therefore, in combination with their inherent electrochemical/fluorescent properties this enables the development of theranostics. ${ }^{107}$ In particular, the 2-D nanomaterial, graphene oxide (GO) has been extensively shown to have inherent antibacterial activity, ${ }^{108}$ by inducing ROS production (oxidative stress) and physical contact with bacteria resulting in damage to their cell membranes. A recent report by Chakraborty and co-workers has explored the physical cutting and oxidative stress mechanisms of both GO and reduced GO (rGO). ${ }^{109}$ The extent of bactericidal efficacy varying between Gram-positive and Gram-negative bacteria was found to be size-, shape-, and typedependent. Gram-positive $S$. aureus was found to be more vulnerable to GO compared to Gram-negative $P$. aeruginosa. Within GO, the oxygen groups enable the wrapping of bacterial cells and inhibits the entry of nutrients. In addition, oxidative stress is generated on the cell membrane for destabilization and disintegration, which leads to leakage of cytoplasmic fluid. A recent study illustrated the significance of GO nanosheets for the treatment of MDR bacteria ("superbugs"). ${ }^{110}$ The MDR bacteria strains included E. coli, K. pneumoniae, S. aureus, $P$. aeruginosa, $P$. mirabilis, and $S$. marcescens. Due to the clinical importance of biofilms, He et al. evaluated the efficacy of GO towards $S$. mutans biofilms, ${ }^{111}$ in which concentration-dependent inhibition was observed for biofilm formation. However, no antibiofilm effect was observed for mature biofilms. This illustrates the need for further optimisation of graphene-based nanomaterials. Consequently, GO platforms were co-loaded with active small molecules and 0-D nanoparticles in order to enhance the antibacterial efficiency via synergistic effects. Motivated to enhance ROS production by GO, Zhou and co-workers loaded the phototherapeutic sodium anthraquinone-2-sulfonate (AQS) onto GO using $\pi-\pi$ interactions to afford the nanocomposite AQS-GO (Fig. 10a). ${ }^{112}$ AQS-GO exhibited light-based inhibitory activity against $E$. coli, where clear cell damage could be seen when compared with GO under irradiation (Fig. 10b). Quaternary ammonium salts (QASs) are well-known antibacterial agents; ${ }^{113,114}$ as such, Ye and co-workers developed a GO nanocomposite using dodecyl dimethyl benzyl ammonium chloride. The QAS (positively charged) was used as a bacteria-targeting unit and an antibacterial agent. ${ }^{115}$ The system provided long-term antibacterial activity against both $E$. coli and $S$. aureus. Other reported strategies aimed at enhancing the therapeutic efficacy of GO include the loading of sodium 1-naphthalenesulfonate onto rGO for chelation of AgNPs, which afforded antibacterial AgNPNA-rGO. ${ }^{116}$ Remarkably, the strategy was superior to AgNP strategies and exhibited long term antibacterial activity with excellent water solubility and low cytotoxicity. The authors proposed that these AgNP-NA-rGO hybrids could be used in

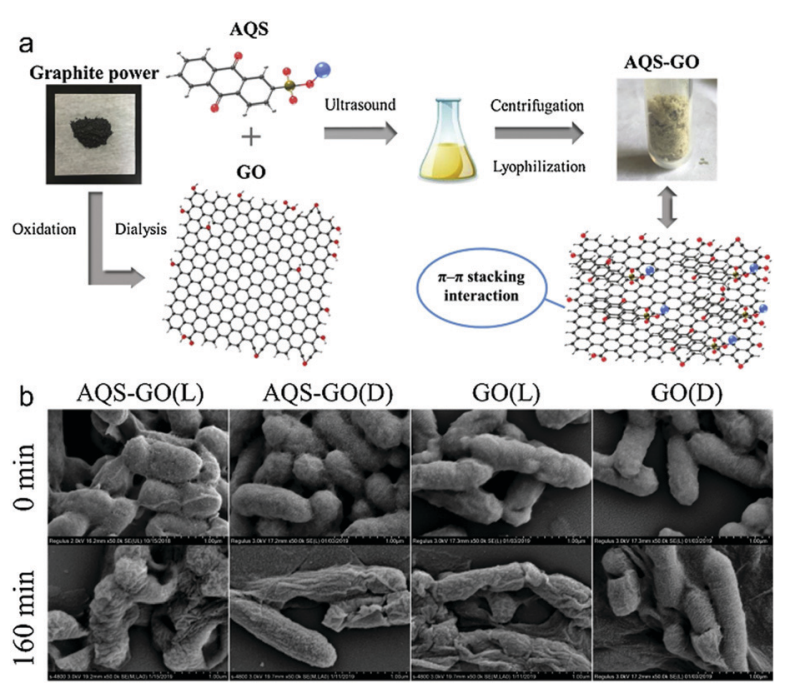

Fig. 10 (a) Schematic illustration of the preparation procedure of a composite antibacterial agent AQS-GO. (b) SEM images of $E$. coli incubated with GO and AQS-GO at 0 min and 160 min under dark (D) and visible light irradiation (L). Images reprinted with permission from ref. 112. Copyright 2019, Elsevier B.V. 
sprayable antibacterial solutions. More recently, Hao and coworkers reported PEG-functionalised GO for the development of stable aqueous AgNP nanocomposites. Non-PEGylated GO with Ag was shown to irreversibly aggregate in aqueous solution, while AgNPs-GO-PEG displayed good aqueous stability, low cytotoxicity and long-term antibacterial activity ( $\sim 95 \%)$ against E. coli and $S$. aureus (stored in saline solution for one week). ${ }^{117}$ Similarly, Zhou and co-workers reported the loading of GO with AgNPs and $\mathrm{CoFe}_{2} \mathrm{O}_{4} \mathrm{NPs}$ for both $\mathrm{Pb}^{2+}$ removal and bacterial absorption/ eradication in contaminated water. ${ }^{118}$

The combination of GO with other nanoplatforms results in systems with therapeutic and diagnostic (visualisation) capability. For example, Kim and co-workers have developed a GO-MoS 2 nanocomposite film through stacking of 2-D $\mathrm{MoS}_{2}$ (molybdenum disulfide) onto GO. ${ }^{119}$ This system exhibited a time-dependent therapeutic effect against $E$. coli, which was visualised using holotomographic (HT) microscopy (Fig. 11). Alternatives to graphene-based materials include graphitic carbon nitride nanosheets, which exhibit antibacterial activities against $E$. coli, $S$. typhimurium, S. enteritidis, $S$. aureus, L. monocytogenes, B. subtilis, and B. cereus. ${ }^{120}$ The use of such graphitic carbon nitride nanosheets is still at the very early stage of development and as such considerably more research is required to evaluate these systems. In summary, the importance of graphene derivatives for biomedical research is attributed to excellent photothermal conversion efficiency, the capacity to co-load active agents, and the satisfactory biocompatibility. However, graphene systems are difficult to completely disperse in aqueous solutions without modification, meaning that other graphene-like 2-D materials are now being considered for development.

After the emergence of GO, a range of other 2D nanomaterials with properties similar to graphene/GO were developed, which are collectively known as graphene-like platforms and exhibit additional functionality. 2-D $\mathrm{MoS}_{2}$ is a popular graphene-like

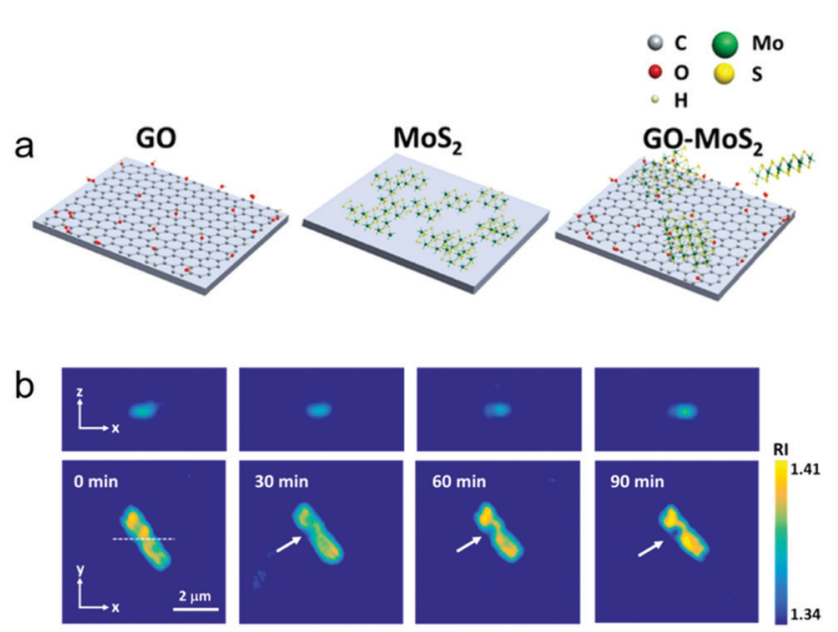

Fig. 11 (a) Schematic illustration of a GO-MoS 2 nanocomposite film consisting of 2-D GO and $\mathrm{MoS}_{2}$. (b) Representative cross-sectional images of 3-D RI tomograms of an E. coli bacterial cell in contact with the $\mathrm{GO}-\mathrm{MoS}_{2}$ surface for $90 \mathrm{~min}$ on the focal plane. Images reprinted with permission from ref. 119. Copyright 2017, American Chemical Society. nanomaterial that has the advantages of graphene and exhibits improved stability/dispersivity. 2-D $\mathrm{MoS}_{2}$ exhibits good structural, physicochemical, optical properties and excellent biocompatibility. ${ }^{121}$ More importantly, $\mathrm{MoS}_{2}$ can be easily metabolized and decomposed in human bodies and is excreted rapidly, which is a significant improvement when compared to graphene derivatives when considering the body damage or side effects induced by these materials. ${ }^{121}$ Recently, Niu et al. reported a Gram-selective antibacterial 2-D $\mathrm{MoS}_{2}$ nanomaterial, which achieved selectivity by controlling the light-irradiation time (photomodulation). ${ }^{122}$ This is of particular significance since providing appropriate treatment for the right bacteria reduces the development of drug-resistant bacteria. As with GO, synergistic studies for the loading of small drug molecules and nanoparticles onto 2-D $\mathrm{MoS}_{2}$ for constructing composites have been performed. A recent report by $\mathrm{Ji}$ and co-workers focused on the combination therapy between antibiotics and PTT. ${ }^{123}$ In this study, ofloxacin (OFLX)-loaded 2-D $\mathrm{MoS}_{2}$ nanoflakes were used as a PTT/antibacterial platform. 2-D $\mathrm{MoS}_{2}$ nanoflakes were modified with electropositive quaternized chitosan (QCS) to improve the aqueous dispersion and act as a targeting moiety for the negatively charged surfaces of bacteria. OFLX-loading on 2-D $\mathrm{MoS}_{2}$ nanoflakes was achieved using $\pi-\pi$ stacking and hydrophobic interactions. Remarkably, QCS- $\mathrm{MoS}_{2}-$ OFLX proved effective as an antibiotic/PTT agent at low temperatures $\left(45{ }^{\circ} \mathrm{C}\right)$ and low antibiotic concentrations. The effectiveness of the 2-D nanomaterial was demonstrated in vitro and in vivo for the treatment of MRSA (Fig. 12). Nitric oxide (NO) is a known signalling molecule found in the human body with inherent antibacterial properties. These properties are

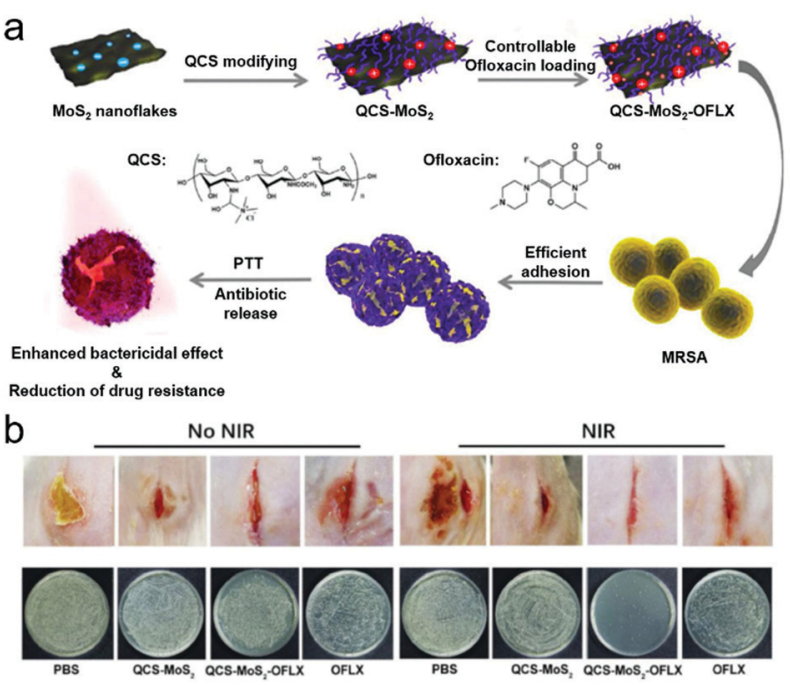

Fig. 12 (a) Schematic illustration of the preparation of QCS-MoS - OFLX for the treatment of methicillin-resistant $S$. aureus (MRSA). (b) Methicillinresistant $S$. aureus-infected wounds in mouse cut models after different treatments of PBS (control), QCS-MoS, QCS $-\mathrm{MoS}_{2}-\mathrm{OFLX}$, and ofloxacin (OFLX) with and without $808 \mathrm{~nm}$ NIR irradiation at day 7. Bacteria were separated from the corresponding wounds and plated on nutrition culture medium. Images reprinted with permission from ref. 123. Copyright 2020, Tsinghua University Press and Springer. 
achieved through the known transformation of NO to more harmful reactive nitrogen species (RNS) i.e., peroxynitrite $\left(\mathrm{ONOO}^{-}\right) \cdot{ }^{124,125}$ Unlike traditional antibiotics, the antibacterial properties of NO are not dependent on the type of bacteria and in addition it is known to promote wound healing. $\mathrm{Gu}$ and coworkers rationalised that the use of a 2-D nanomaterial would facilitate the effective delivery of NO (nanovehicle) and improve the therapeutic effects against bacteria. As a result, $\alpha$-CD modified 2-D $\mathrm{MoS}_{2}$ nanosheets were assembled with the heat sensitive (NO) donor $N, N^{\prime}$-di-sec-butyl- $N, N^{\prime}$-dinitroso-1, 4-phenylenediamine (BNN6). ${ }^{126}$ NIR light irradiation (808 nm) activated PTT and generated NO. The antibacterial effects were successfully demonstrated using infected mouse models.

2D $\mathrm{MoS}_{2}$ has been used for loading nanoparticles, in combination with AgNPs and D-Cys for the construction of an effective antibacterial nanocomposite. ${ }^{127}$ More recently, a multifunctional nanomaterial was developed consisting of 2-D $\mathrm{MoS}_{2}$ nanosheets and AgBr nanoparticles (AgBr@MoS 2 ) grown on Ti-based implant materials; the $\mathrm{AgBr}$ was co-loaded to reduce the inherent toxicity. Visible light irradiation $(660 \mathrm{~nm})$ of the nanomaterial resulted in significant phototherapeutic efficacy against $E$. coli and $S$. aureus. ${ }^{128} 2-\mathrm{D} \mathrm{Ti}_{3} \mathrm{C}_{2} \mathrm{~T}_{x}$ MXene nanosheets have emerged as an attractive nanomaterial for biomedical applications. ${ }^{129}$ In 2016, $\mathrm{Ti}_{3} \mathrm{C}_{2} \mathrm{~T}_{x}$ MXene nanosheets were evaluated for their antibacterial properties and compared with GO. Significantly better antibacterial effects were observed when compared to those of GO $(\sim 2-4$ fold greater) against both $E$. coli and B. subtilis. ${ }^{130}$ As with graphene, SEM and TEM images revealed physical damage to the cell membrane and biological assays identified the induction of oxidative stress. The authors anticipate that MXenes will find use in biofouling and bactericidal applications due to the good biocompatibility. Recently, Zhang and co-workers used the known low-cost and biocompatible semi-conductor material, $\mathrm{Sb}_{2} \mathrm{Se}_{3}$ for the development of an antibacterial nanomaterial. ${ }^{131}$ In this report, polyvinylpyrrolidone (PVP)-capped $\mathrm{Sb}_{2} \mathrm{Se}_{3}$ was used for the treatment of bacteria including $E$. coli and methicillin-resistant $S$. aureus (MRSA) in vivo. 2-D tungsten disulfide $\left(\mathrm{WS}_{2}\right)$ nanomaterials have been explored for water filtration applications, exhibiting excellent antibacterial properties against $S$. aureus and $E$. coli. ${ }^{132}$ More recently, Pramanik and co-workers developed a combined 2-D and 0-D nanomaterial strategy for the identification of antibiotic-resistant bacteria. ${ }^{133}$ This was achieved through the construction of $\mathrm{WS}_{2}-\mathrm{AuNPs}$ and using the surface enhanced Raman spectroscopic properties of AuNPs enabling the rapid detection (90 min) of MDR Salmonella DT104. Ikram and co-workers have reported Zr-doped $\mathrm{MoS}_{2}$ nanosheets for catalytic and antibacterial applications. ${ }^{134}$ These systems were effective against $E$. coli and $S$. aureus through the catalytic generation of ROS. This catalytic-based antibacterial approach was then further elucidated by Yin and coworkers by directly loading enzymes onto 2-D $\mathrm{MoS}_{2}$. They reported lysozyme-coated $\mathrm{MoS}_{2}$ nanosheets $\left(\right.$ Lys- $\mathrm{MoS}_{2}$ ), which displayed good antibacterial efficacy against Gram-negative E. coli and Gram-positive B. subtilis. ${ }^{135}$ This was due to synergistic effects between the lysozyme and peroxidase activity of the $\mathrm{MoS}_{2}$ nanosheets.

Often medical implants are prone to bacterial infection; for this reason, Wu and co-workers developed a chitosan@ $\mathrm{MoS}_{2}$ nanocomposite to overcome this problem. Chitosan@ $\mathrm{MoS}_{2}$ was deposited onto medical Ti-based implants through an electrophoretic deposition method. ${ }^{136}$ Upon dual-light irradiation of chitosan@ $\mathrm{MoS}_{2}-\mathrm{Ti}(660 \mathrm{~nm}$ and $808 \mathrm{~nm})$, significant bacterial inhibition was observed in vitro and in vivo. Moreover, 2-D $\mathrm{MoS}_{2}$ could be combined with graphene derivatives. PEG-2-D $\mathrm{MoS}_{2} / \mathrm{rGO}$ therapeutic nanoflakes were developed by Li and co-workers and loaded with a streptomycin sulfate (SS) antibiotic. In combination with NIR light irradiation, the therapeutic efficacy of PEG-MoS $2 /$ rGO-SS was significantly enhanced and a synergistic therapeutic effect was observed between SS and light-based therapy. ${ }^{137}$ Black phosphorus (BP) is an emerging 2-D nanomaterial used for semiconductor applications. Aksoy and co-workers reported $\mathrm{BP} / \mathrm{AuNP}$ nanocomposites for antibacterial applications with good biocompatibility. ${ }^{138}$ The NIR laser irradiation (808 NM) of these nanocomposites resulted in the PTT/PDT treatment of planktonic bacteria and biofilm-based E. faecalis (Fig. 13a). Live/dead staining and fluorescence imaging were used to evaluate the therapeutic efficacy of the $\mathrm{BP} / \mathrm{Au}$ nanocomposites against E. faecalis (Fig. 13b). For more examples of nanomaterials beyond graphene (NBG), the reader is directed to an excellent review by Yin and $\mathrm{Gu}^{106}$

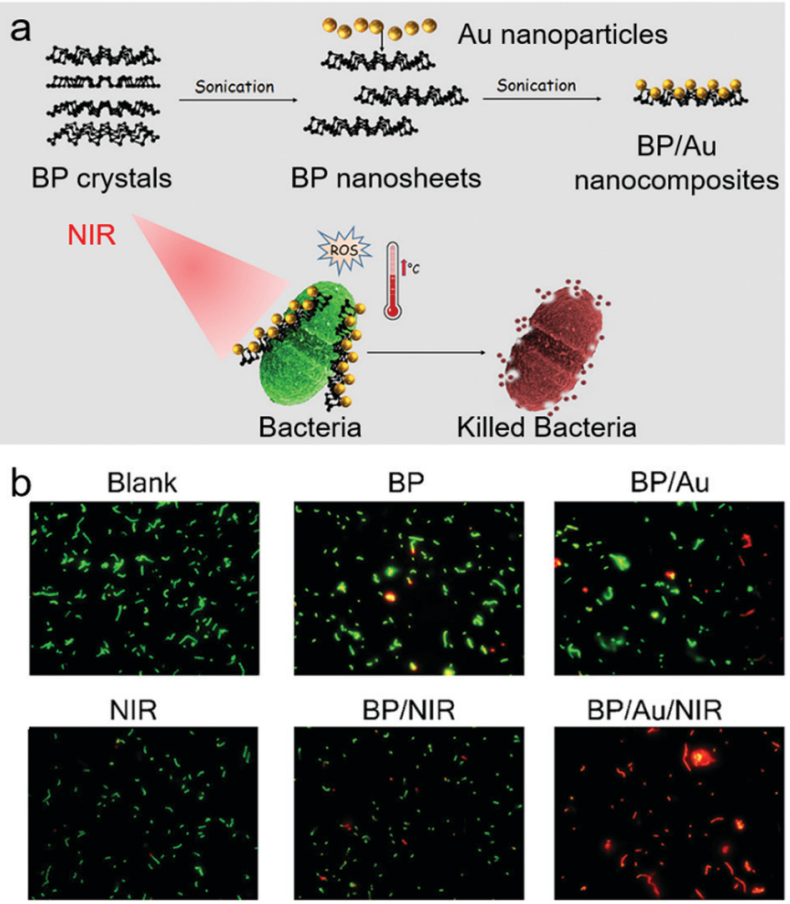

Fig. 13 (a) Schematic illustration of the construction process of a black phosphorus- and AuNP-based nanocomposite BP/Au and its effects in treating bacteria upon NIR irradiation. (b) Fluorescence images of E. faecalis cells after live/dead staining with different treatment protocols. Images reprinted with permission from ref. 138. Copyright 2020, American Chemical Society. 


\section{3-D and other high-level nanomaterials}

Compared to 0-D, 1-D, and 2-D nanomaterials, systems with a comprehensive 3-D or 3-D-like structure are only beginning to be used for biomedical applications. 3-D nanomaterials are materials that are not confined to the nanoscale in any dimension, these include nanoballs (dendritic structures), nanocoils, nanoclusters, nanocones, nanopillars and nanoflowers. ${ }^{139,140}$ In addition, 3-D-like nanomaterials can be formed by the assembly of $0-\mathrm{D}, 1-\mathrm{D}$, and 2-D nanomaterials. Moreover, there are some highly-complex composite materials with advanced supramolecular structure, which cannot be defined as 0-D, 1-D, or 2-D materials; so, they are also included and discussed here. Two of the most prominent skeleton-like nanomaterials are metal-organic frameworks (MOFs) and covalent organic frameworks (COFs), which are generally biocompatible towards tissues. ${ }^{141,142}$ These are porous materials formed through the self-assembly of covalent bonds (COFs) or through the coordination between metal ions and multidentate ligands (MOFs). While both 2-D structured and 3-D structured MOFs and COFs are available, in particular 3-D structured ones (3-D MOFs/COFs) exhibit considerable antibacterial activity. ${ }^{141,142}$ In 2020, Qu and co-workers reported the construction of a MOF@COF hybrid that exhibited nanozyme characteristics (peroxidasebased) for the treatment of bacteria. This was the first example where COFs were used for tuning the catalytic and therapeutic performance of MOFs, resulting in the effective treatment of both $E$. coli and $S$. aureus. ${ }^{143}$ Mesoporous-based materials have been used to form fine microstructures. Examples include a mesostructured, Ag containing, silica-based calcium phosphate (80SiO2-CaO-P2O5 $+\mathrm{Ag}$ ) and bioactive ceramic powders $(\mathrm{Ag} /$ 80S) reported by Shih and co-workers. ${ }^{144}$ With the help of Ag as a therapeutic agent and the outside shell as a carrier, Ag/80S was shown to inhibit MDR bacteria, while acting as a bone graft material with osteoinductive and osteoconductive properties. In addition, Peng and co-workers developed an antibacterial polymer based on mesoporous bioactive glass (MBG) for bone material applications. ${ }^{145}$ MBG was first modified with dopamine, which underwent oxidative self-polymerization to form pMBG, which bound and reduced $\mathrm{Ag}^{+}$ions to metallic $\mathrm{Ag}$ (Fig. 14a). Ag@pMBG was incorporated into a polymer scaffold (PLLA-PGA) to provide long-term antibacterial activity against $E$. coli with good cytocompatibility, which can only be ensured with the presence of incorporated Ag (Fig. 14b). A novel "nanocube" was developed by Liu and co-workers, where a zeolitic imidazolate framework (ZIF-8) was pyrolyzed at $800{ }^{\circ} \mathrm{C}$ to form an NCS (nanocube-like structure) followed by the adhesion of silver ions to the surface of the NCSs by sonication in ethanol. ${ }^{146}$ With the addition of sulphur, the metal ions can in situ form small metal chalcogenides on the surface of the NCSs. Both PDT and PTT effects were observed with good NIR response and low cytotoxicity. $S$. aureus was effectively treated using excitation by an $808 \mathrm{~nm}$ laser for $20 \mathrm{~min}$. A $\mathrm{TiO}_{2}$ nanorod array was developed by Zhang and co-workers in 2021, with multiple mechanisms including PDT, PTT, and physical killing

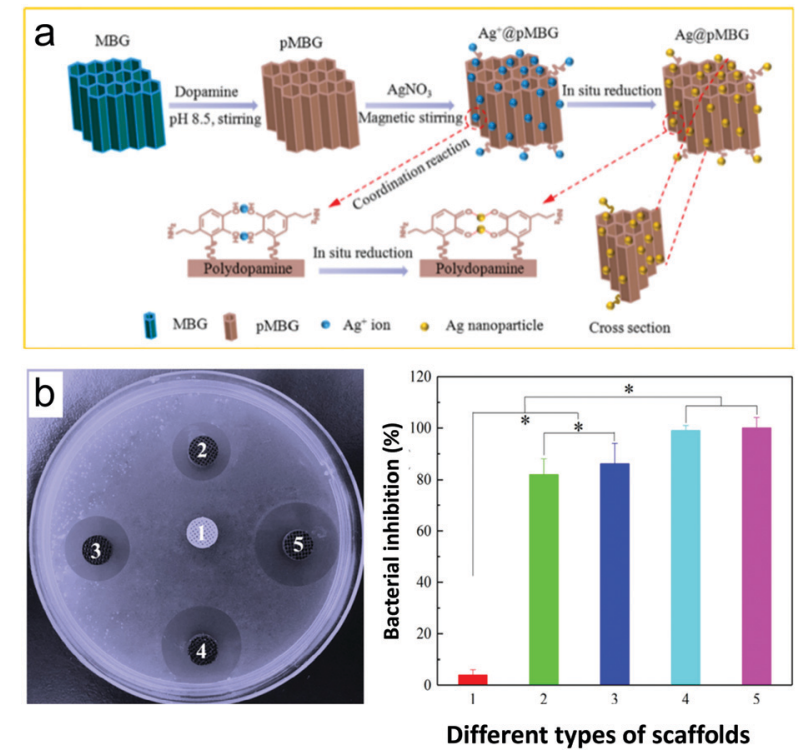

Fig. 14 (a) Schematic illustration of the preparation of an antibacterial polymer scaffold based on mesoporous bioactive glass (MBG). (b) E. coli inhibition rings and inhibition rates of PLLA-PGA/MBG (1), PLLA-PGA/ 2Ag@pMBG (2), PLLA-PGA/4Ag@apBG (3), PLLA-PGA/6Ag@pMBG (4), and PLLA-PGA/8Ag@PMBG (5) after $24 \mathrm{~h}$ of culture. Images reprinted with permission from ref. 145. Copyright 2020, Elsevier B.V.

to produce excellent antibacterial properties on titanium towards $E$. coli and $S$. aureus after $15 \mathrm{~min}$ of $808 \mathrm{~nm}$ irradiation. ${ }^{147}$ The system was applied for in vivo antibacterial treatment including in mouse tissue infection models and the bone tissue around the $\mathrm{Ti}$ implants. Moreover, this nanorod array was found to improve new bone formation around implants.

Ag-decorated polydopamine/mesoporous silica composites were constructed to enhance the therapeutic efficiency of $\mathrm{Ag}$ and reduce toxicity via an incorporating/releasing effect triggered by $\mathrm{pH}$ changes and ROS, and was used for the treatment of drugresistant bacteria and tumor cells. ${ }^{148}$ Mesostructured cellular silica foams (MCF) were demonstrated by Xia and co-workers as excellent antibacterial hemostatic agents. ${ }^{149}$ Metallic glass consisting of $\mathrm{Mg}, \mathrm{Ag}$, and $\mathrm{Cu}$ has been reported by Wang and co-workers for the long-term treatment of $S$. aureus and E. coli with the sustainable release of $\mathrm{Cu}$ and $\mathrm{Ag}$ ions. ${ }^{150}$ Compared with all the individual components, this system was shown to perform significantly better due to synergistic effects. Other strategies include the development of 3-D-based graphene derivatives for antibacterial applications. For example, tannic acid was used to reduce GO and induce self-assembly to form a graphene hydrogel, ${ }^{151}$ which represents an environmentallyfriendly, and cost effective approach to treat both $S$. aureus and E. coli. The 3-D graphene exhibited high porosity, low density, hydrophobicity, good mechanical performance and thermal stability compared with normal 2-D graphene, with the ability to adsorb a range of oils, dyes and organic solvents. These results exhibited promise for water purification applications with the ability to treat $S$. aureus and E. coli. ${ }^{152}$ Bahadur and co-workers developed a 3-D layered double nanohybrid consisting of $\mathrm{Mg}-\mathrm{Al}$ layered double hydroxide-reduced GO (rGO). The 


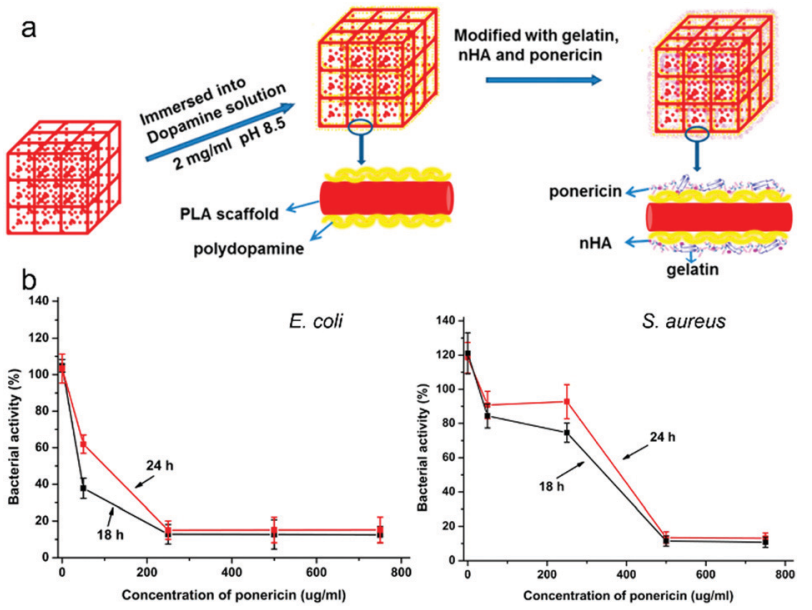

Fig. 15 (a) Schematic illustration of a composite 3-D-printed polylactic acid (PLA) scaffold. (b) Antibacterial effects of the ponericin-modified scaffold against E. coli and S. aureus at different ponericin concentrations. Images reprinted with permission from ref. 155. Copyright 2017, American Chemical Society.

synergistic antibacterial effect observed against $E$. coli was much better than those for the individual components with enhanced selectivity, which was attributed to protein degradation and GSH loss through the induction of oxidative stress. ${ }^{153}$ Moreover, a vertical heterostructure consisting of $\mathrm{MoS}_{2}$-coated rGO was developed by $\mathrm{Yu}$ and co-workers using a microwave-assisted hydrothermal method, which exhibited in situ bacterial binding with enhanced nanozyme activity and producing ROS for excellent $E$. coli/ $S$. aureus antibacterial effect. ${ }^{154}$ The overall effects observed within the heterostructure were synergistically improved compared with those of single $\mathrm{MoS}_{2}$ or rGO. The mechanism was relevant for the catalytic generation of a range of ROS including hydroxyl radicals $\left(\mathrm{OH}^{\bullet}\right)$ from $\mathrm{H}_{2} \mathrm{O}_{2}$ by the nanozyme upon light activation, making the system suitable for use in mouse wound-infection models.

Huang and co-workers reported a degradable 3-D-printed polylactic acid (PLA) scaffold for applications in bone tissue engineering. ${ }^{155}$ The PLA scaffold was elaborated around a 3-Dprinted core functionalized with an adherent polydopamine coating, and the modified scaffold was then used to immobilize a mixture of gelatin, needle-like nanohydroxyapatite (nHA), and ponericin (Fig. 15a). Long-term antibacterial activity was observed towards E. coli and S. aureus (Fig. 15b). Zheng and co-workers developed AgNP-associated carbon aerogels p-BC/ AgNPs for antibacterial applications. ${ }^{156} \mathrm{p}$-BC/AgNPs exhibited excellent antibacterial efficiency against $E$. coli and $S$. aureus, which was better than with just AgNPs and p-BC. Moreover, p-BC/AgNPs exhibited excellent cell attachment and biocompatibility. Due to these properties, the authors proposed that this newly developed antibacterial material could be used for wound dressings, medical implants, and drug release applications.

\section{Conclusions}

Bacterial infections are a major health concern, threatening the lives of millions of people worldwide. Without effective treatments, a wide range of serious health complications will develop, including high mortality rates. ${ }^{3}$ The emergence of MDR "superbugs" is now becoming more and more problematic in clinical scenarios. The use of functional materials represents an exciting opportunity for bacterial treatments due to their outstanding PDT/PTT properties and drug carrier (loading/releasing) properties, which provides an opportunity to reduce the risks of developing drug-resistant bacteria. ${ }^{157}$ Currently, the major focus of nanomaterials research has been towards anticancer applications. The antibacterial applications of nanomaterials started a decade ago, using them as functional building blocks for the fabrication of antibacterial surfaces. ${ }^{158,159}$ Graphene-based antibacterial papers were initially developed where macroscopic GO and rGO papers can be conveniently fabricated from their suspension through simple vacuum filtration, and were found to inhibit the growth of $E$. coli. ${ }^{160}$ A new approach to fabricate antibacterial cotton fabrics by attaching single layer GO has also been reported, and the properties of GO remained even after repeated washing (ca. 100 times). Appropriate levels of crosslinking can be induced both by radiation and by chemical methods. Compared with single cotton or GO, the composite system formed by cotton and GO has an improved antibacterial effect towards E. coli and B. subtilis. ${ }^{161}$ In this review, we provide an insight into recent research that has focused on the use of functional nanomaterials for the treatment of bacteria. The research covered in this review has been organised according to the materials dimensions, clearly highlighting the importance of the morphologies of the nanomaterials used.

Despite the many successful experiments demonstrating the use of these systems, their use for clinical applications still represents a major hurdle due to their instability and potential side-toxicity in vivo. As such this has significantly prevented their development into commercially available therapeutics, which is further exemplified by the scarcity of functional materials being evaluated in vivo. ${ }^{162}$ However, the most recent in vivo studies have demonstrated excellent therapeutic efficacy without any serious toxicity being observed. Possible methods for enhancing biocompatibility may include functionalisation of the nanomaterial platforms with carbohydrates and peptides to afford targeted systems. Furthermore, these biomolecules may provide additional antibacterial properties. A representative example is polymeric antimicrobial peptides (AMPs) that have been recently developed by Liu and co-workers, ${ }^{163-165}$ which are cationic $^{163,164}$ and amphiphilic ${ }^{165}$ and exhibit antifungal activity, ${ }^{163}$ immunomodulatory functions, ${ }^{165}$ and significant time-resolved effects against multiple bacterial species and biofilms both in vivo and for mouse infection models. In the near future, nanomaterials emerging from the developments in materials science, will result in improved clinically relevant properties such as stability, biocompatibility, and biodegradability, and result in more tissue-friendly targeting/assisting agents. With this review we have summarized the current state of the art for nanomaterial-based antibacterial systems and provided guidelines for the development of new and improved nanomaterial platforms. We are confident that with sustained hard work in this area, ${ }^{166}$ 
an antibacterial nanomaterial system could soon be available to treat patients with MDR infections.

\section{Abbreviations}

E. coli

S. aureus

MRSA

P. aeruginosa

S. epidermidis

P. gingivalis

P. micros

P. intermedia

S. mutans

B. subtilis

A. baumannii

S. faecalis

E. cloacae

B. pertussis

C. albicans

K. pneumoniae

P. mirabilis

S. marcescens

DDS

PDT

PTT

Ag

Ti

$\mathrm{Cu}$

Pd

Se

ROS

$0-\mathrm{D}$

$1-\mathrm{D}$

2-D

3-D

GQDs

AgNPs

NCC

D-Cys-AgNPs

EPS

D-Cys

$\mathrm{Bi}$

BiNPs

$\gamma$-CD

MOFs

GRGDs

PVA

CNDs

OD

MC3T3-E1 cells

CA

CDs

GO

TEM
Escherichia coli

Staphylococcus aureus

Methicillin-resistant $S$. aureus

Pseudomonas aeruginosa

Staphylococcus epidermidis

Porphyromonas gingivalis

Peptostreptococcus micros

Prevotella intermedia

Streptococcus mutans

Bacillus subtilis

Acinetobacter baumannii

Streptococcus faecalis

Enterobacter cloacae

Bordetella pertussis

Candida albicans

Klebsiella pneumoniae

Proteus mirabilis

Serratia marcescens

Drug delivery system

Photodynamic therapy

Photothermal therapy

Silver

Titanium

Copper

Palladium

Selenium

Reactive oxygen species

Zero-dimensional

One-dimensional

Two-dimensional

Three-dimensional

Graphene quantum dots

Silver nanoparticles

Nanocrystalline cellulose

D-Cysteine functionalised AgNPs

Extracellular polymeric substances

D-Cysteine

Bismuth

Bismuth nanoparticles

$\gamma$-Cyclodextrin

Metal-organic frameworks

Gly-Arg-Gly-Asp-Ser

Poly(vinyl alcohol)

Carbon nanodots

Optical density

Mouse embryo osteoblast precursor cells

Citric acid

Carbon dots

Graphene oxide

Transmission electron microscopy
SEM

AuNPs

DAPT

DGNPS

uDGNPs

$\mathrm{MOF} @ \mathrm{CeO}_{2} \mathrm{NPs}$

ATP

Ce

$\mathrm{Zr}$

MEH-PPV

BFPB

TMB

CEMA

HEMA

SWNTs

MWCNTS

PES

UF

GNRs

PEO

NIR

Pp4N

AgNWs

4MPFG

HBTC

AuNRs

MMO

rGO

AgNP-NA-rGO

AQS

PEG

$\mathrm{MoS}_{2}$

HT

MDR

QAS

NBG

RI

SS

OFLX

QCS

NO

RNS

$\mathrm{ONOO}^{-}$

BNN6

Lys-MoS

PVP

$\mathrm{WS}_{2}$

$\mathrm{BP}$

COFs

MBG
Scanning electron microscopy

Gold nanoparticles

4,6-diamino-2-pyrimidinethiol

(4,6-Diamino-2-pyrimidinethiol)-modified

gold nanoparticles

Ultrasmall DGNPs

$\mathrm{CeO}_{2}$-decorated nanoparticle MOFs

Adenosine triphosphate

Cerium

Zirconium

2-Methoxy-5-((2-ethylhexyl)oxy)-p-

phenylenevinylene

4-\{2,2-Bis[(4-formylphenoxy)methyl]-3-(4-

formylphenoxy)propoxy\}benzaldehyde

$3,3^{\prime}, 5,5^{\prime}$-Tetramethylbenzidine

2-Chloroethyl methacrylate

Hydroxyethyl methacrylate

Single-walled nanotubes

Multi-walled carbon nanotubes

Polyethersulfone

Ultrafiltration

Graphene nanoribbons

Poly(ethylene oxide)

Near-infrared

Polycationic porphyrin

Nanowires

$N, N$-Bis(pyridyl-4-methyl)- $N$-fluorenyl-9-

methoxycarbonyl (Fmoc)-L-glutamate

1,3,5-Benzenetricarboxylic acid

Gold nanorods

Mixed metal oxide

Reduced GO

AgNP/sodium 1-naphthalenesulfonate-

functionalized reduced graphene oxide

Anthraquinone-2-sulfonate

Polyethylene glycol

Molybdenum disulfide

Holotomographic

Multidrug-resistance

Quaternary ammonium salt

Nanomaterials beyond graphene

Refractive index

Streptomycin sulfate

Ofloxacin

Quaternized chitosan

Nitric oxide

Reactive nitrogen species

Peroxynitrite

$N, N^{\prime}$-Di-sec-butyl- $N, N^{\prime}$-dinitroso-1,4-

phenylenediamine

Lysozyme-coated $\mathrm{MoS}_{2}$ nanosheets

Polyvinyl pyrrolidone

Tungsten disulfide

Black phosphorus

Covalent organic frameworks

Mesoporous bioactive glass 
pMBG

Self-polymerization mesoporous bioactive glass

PGA Polyglycolic acid

ZIF Zeolitic imidazolate framework

NCS Nanocube-like structure

MCF

GSH

$\mathrm{OH}^{\bullet}$

PLA

nHA

AMPs

Mesostructured cellular silica foam

Glutathione

Hydroxyl radicals

Polylactic acid

Needle-like nanohydroxyapatite

Antimicrobial peptides

\section{Conflicts of interest}

The authors declare no conflicts of interest.

\section{Acknowledgements}

The authors thank the National Natural Science Foundation of China (No. 21788102, 91853201, 21907030, 81872775 and 21776078), the National Science and Technology Major Project of China (No. 2018ZX10732202 and 2018ZX10302205), the Shanghai Municipal Science and Technology Major Project (No. 2018SHZDZX03), the International Cooperation Program of Shanghai Science and Technology Committee (No. 17520750100) and the Fundamental Research Funds for the Central Universities (222201717003) for financial support. TDJ wishes to thank the Royal Society for a Wolfson Research Merit Award and the Open Research Fund of the School of Chemistry and Chemical Engineering, Henan Normal University for support (2020ZD01).

\section{Notes and references}

1 J. W. Miller, V. Hanson, G. D. Johnson, J. E. Royalty and L. C. Richardson, From cancer screening to treatment: Service delivery and referral in the national breast and cervical cancer early detection program, Cancer, 2014, 120, 2549-2556.

2 M. Liu, F. O. Fields, J. S. Prescott, A. Bello, N. Bower, S. Darakjy, J. Hartke, V. Kadambi, D. Lapadula, A. Stoch and M. Derzi, Evaluation of therapeutics for severely debilitating or life-threatening diseases or conditions: Defining scope to enable global guidance development, Clin. Pharmacol. Ther., 2020, 107, 514-520.

3 S. Sakanaka and Y. Okada, Inhibitory effects of green tea polyphenols on the production of a virulence factor of the periodontal-disease-causing anaerobic bacterium Porphyromonas gingivalis, J. Agric. Food Chem., 2004, 52, 1688-1692.

4 V. Bhandari, K. S. Wong, J. L. Zhou, M. F. Mabanglo, R. A. Barey and W. A. Houry, The role of ClpP protease in bacterial pathogenesis and human diseases, ACS Chem. Biol., 2018, 13, 1413-1425.
5 A. A. Karbelkar and A. L. Furst, Electrochemical diagnostics for bacterial infectious diseases, ACS Infect. Dis., 2020, 6, 1567-1571.

6 C. S. Higgins, S. M. Murtough, E. Williamson, S. J. Hiom, D. J. Payne, A. D. Russell and T. R. Walsh, Resistance to antibiotics and biocides among non-fermenting Gramnegative bacteria, Clin. Microbiol. Infect., 2001, 7, 308-315.

7 A. R. M. Coates and Y. Hu, Novel approaches to developing new antibiotics for bacterial infections, Br. J. Pharmacol., 2007, 152, 1147-1154.

8 R. Jijie, A. Barras, F. Teodorescu, R. Boukherroub and S. Szunerits, Advancements on the molecular design of nanoantibiotics: Current level of development and future challenges, Mol. Syst. Des. Eng., 2017, 2, 349-369.

9 V. Yarlagadda, G. B. Manjunath, P. Sarkar, P. Akkapeddi, K. Paramanandham, B. R. Shome, R. Ravikumar and J. Haldar, Glycopeptide antibiotic to overcome the intrinsic resistance of Gram-negative bacteria, ACS Infect. Dis., 2016, 2, 132-139.

10 M. A. Abedalwafa, Y. Li, C. Ni and L. Wang, Colorimetric sensor arrays for the detection and identification of antibiotics, Anal. Methods, 2019, 11, 2836-2854.

11 C. Schnaars, G. Kildahl-Andersen, A. Prandina, R. Popal, S. Radix, M. L. Borgne, T. Gjøen, A. M. S. Andresen, A. Heikal, O. A. Økstad, C. Fröhlich, Ø. Samuelsen, S. Lauksund, L. P. Jordheim, P. Rongved and O. A. H. Åstrand, Synthesis and preclinical evaluation of TPA-based zinc chelators as metallo- $\beta$-lactamase inhibitors, ACS Infect. Dis., 2018, 4, 1407-1422.

12 Y. Liu, M. Song, S. Ding and K. Zhu, Discovery of linear low-cationic peptides to target methicillin-resistant staphylococcus aureus in vivo, ACS Infect. Dis., 2019, 5, 123-130.

13 C. S. Vinagreiro, A. Zangirolami, F. A. Schaberle, S. C. C. Nunes, K. C. Bianco, N. M. Inada, G. J. Silva, A. A. C. C. Pais, V. S. Bagnato, L. G. Arnaut and M. M. Pereira, Antibacterial photodynamic inactivation of antibiotic-resistant bacteria and biofilms with nanomolar photosensitizer concentrations, ACS Infect. Dis., 2020, 6, 1517-1526.

14 K. R. V. Thappeta, Y. S. Vikhe, A. M. H. Yong, M. B. ChanPark and K. A. Kline, Combined efficacy of an antimicrobial cationic peptide polymer with conventional antibiotics to combat multidrug-resistant pathogens, ACS Infect. Dis., 2020, 6, 1228-1237.

15 T.-K. Nguyen, S. J. Lam, K. K. K. Ho, N. Kumar, G. G. Qiao, S. Egan, C. Boyer and E. H. H. Wong, Rational design of single-chain polymeric nanoparticles that kill planktonic and biofilm bacteria, ACS Infect. Dis., 2017, 3, 237-248.

16 L. J. Stephens, M. V. Werrett, A. C. Sedgwick, S. D. Bull and P. C. Andrews, Antimicrobial innovation: a current update and perspective on the antibiotic drug development pipeline, Future Med. Chem., 2020, 12, 2035-2065.

17 C. Wu, L. Fang, X. Huang and P. Jiang, Three-dimensional highly conductive graphene-silver nanowire hybrid foams for flexible and stretchable conductors, ACS Appl. Mater. Interfaces, 2014, 6, 21026-21034. 
18 E. H. Huisman, A. G. Shulga, P. J. Zomer, N. Tombros, D. Bartesaghi, S. Z. Bisri, M. A. Loi, L. J. A. Koster and B. J. Wees, High gain hybrid graphene-organic semiconductor phototransistors, ACS Appl. Mater. Interfaces, 2015, 7, 11083-11088.

19 W. G. Chong, J.-Q. Huang, Z.-L. Xu, X. Qin, X. Wang and J.-K. Kim, Lithium-sulfur battery cable made from ultralight, flexible graphene/carbon nanotube/sulfur composite fibers, Adv. Funct. Mater., 2017, 27, 1604815.

20 H. Liang, W. Shuang, Y. Zhang, S. Chao, H. Han, X. Wang, H. Zhang and L. Yang, Graphene-like multilayered CuS nanosheets assembled into flower-like microspheres and their electrocatalytic oxygen evolution properties, ChemElectroChem, 2018, 5, 494-500.

21 F. Rasch, F. Schütt, L. M. Saure, S. Kaps, J. Strobel, O. Polonskyi, A. S. Nia, M. R. Lohe, Y. K. Mishra, F. Faupel, L. Kienle, X. Feng and R. Adelung, Wetchemical assembly of $2 \mathrm{D}$ nanomaterials into lightweight, microtube-shaped, and macroscopic 3D networks, ACS Appl. Mater. Interfaces, 2019, 11, 44652-44663.

$22 \mathrm{D}$. Hu and W. Ma, Nanocellulose as a sustainable building block to construct eco-friendly thermally conductive composites, Ind. Eng. Chem. Res., 2020, 59, 19465-19484.

23 G. Zhou, E. Goshi and Q. He, Micro/nanomaterialsaugmented hydrogen therapy, Adv. Healthcare, Mater., 2019, 8, 1900463.

24 G.-B. Qi, Y.-J. Gao, L. Wang and H. Wang, Self-assembled peptide-based nanomaterials for biomedical imaging and therapy, Adv. Mater., 2018, 30, 1703444.

25 J. Bourquin, A. Milosevic, D. Hauser, R. Lehner, F. Blank, A. Petri-Fink and B. Rothen-Rutishauser, Biodistribution, clearance, and long-term fate of clinically relevant nanomaterials, Adv. Mater., 2018, 30, 1704307.

26 M. Li, Z. Luo and Y. Zhao, Hybrid nanoparticles as drug carriers for controlled chemotherapy of cancer, Chem. Rec., 2016, 16, 1833-1851.

27 N. Gong, Y. Zhang, Z. Zhang, X. Li and X.-J. Liang, Functional nanomaterials optimized to circumvent tumor immunological tolerance, Adv. Funct. Mater., 2019, 29, 1806087.

28 Y.-C. Yeh, T.-H. Huang, S.-C. Yang, C.-C. Chen and J.-Y. Fang, Nano-based drug delivery or targeting to eradicate bacteria for infection mitigation: A review of recent advances, Front. Chem., 2020, 8, 286-308.

29 H. Zheng, R. Ma, M. Gao, X. Tian, Y.-Q. Li, L. Zeng and R. Li, Antibacterial applications of graphene oxides: Structure-activity relationships, molecular initiating events and biosafety, Sci. Bull., 2018, 63, 133-142.

30 Y. Cai, P. Liang, Q. Tang, X. Yang, W. Si, Q. Zhang and X. Dong, Diketopyrrolopyrrole-triphenylamine organic nanoparticles as multifunctional reagents for photoacoustic imaging-guided photodynamic/photothermal synergistic tumor therapy, ACS Nano, 2017, 11, 1054-1063.

31 Z.-C. Xiong, Z.-Y. Yang, Y.-J. Zhu, F.-F. Chen, Y.-G. Zhang and R.-L. Yang, Ultralong hydroxyapatite nanowires-based paper co-loaded with silver nanoparticles and antibiotic for long-term antibacterial benefit, ACS Appl. Mater. Interfaces, 2017, 9, 22212-22222.

32 K. B. William and V. T. Christoph, Neurotoxicology of Nanomaterials, Chem. Res. Toxicol., 2020, 33, 1121-1144.

33 P. Makvandi, C.-Y. Wang, E. N. Zare, A. Borzacchiello, L.-N. Niu and F. R. Tay, Metal-based nanomaterials in biomedical applications: Antimicrobial activity and cytotoxicity aspects, Adv. Funct. Mater., 2020, 30, 1910021.

34 S. Cheeseman, A. J. Christofferson, R. Kariuki, D. Cozzolino, T. Daeneke, R. J. Crawford, V. K. Truong, J. Chapman and A. Elbourne, Antimicrobial metal nanomaterials: From passive to stimuli-activated applications, Adv. Sci., 2020, 7, 1902913.

35 Y. Wang, Y. N. Yang, Y. Shi, H. Song and C. Z. Yu, Antibiotic-free antibacterial strategies enabled by nanomaterials: Progress and perspectives, Adv. Mater., 2019, 32, 1904106.

36 Y. Tao, Y. H. Lin, Z. Z. Huang, J. S. Ren and X. G. Qu, Incorporating graphene oxide and gold nanoclusters: A synergistic catalyst with surprisingly high peroxidase-like activity over a broad $\mathrm{pH}$ range and its application for cancer cell detection, Adv. Mater., 2013, 25, 2594-2599.

37 L. Shi, J. G. Chen, L. J. Teng, L. Wang, G. L. Zhu, S. Liu, Z. T. Luo, X. T. Shi, Y. J. Wang and L. Ren, The antibacterial applications of graphene and its derivatives, Small, 2016, 12, 4165-4184.

38 T. Liu, C. Wang, X. Gu, H. Gong, L. Cheng, X. Z. Shi, L. Z. Feng, B. Q. Sun and Z. Liu, Drug delivery with PEGylated MoS2 nano-sheets for combined photothermal and chemotherapy of cancer, Adv. Mater., 2014, 26, 3433-3440.

39 J. Y. Han, H. P. Xia, Y. F. Wu, S. N. Kong, A. Deivasigamani, R. Xu, K. M. Hui and Y. J. Kang, Single-layer MoS2 nanosheet grafted upconversion nanoparticles for nearinfrared fluorescence imaging-guided deep tissue cancer phototherapy, Nanoscale, 2016, 8, 7861-7865.

40 H.-Z. Lai, W.-Y. Chen, C.-Y. Wu and Y.-C. Chen, Potent antibacterial nanoparticles for pathogenic bacteria, ACS Appl. Mater. Interfaces, 2015, 7, 2046-2054.

41 Y. Qiao, F. Ma, C. Liu, B. Zhou, Q. Wei, W. Li, D. Zhong, Y. Li and M. Zhou, Near-infrared laser-excited nanoparticles to eradicate multidrug-resistant bacteria and promote wound healing, ACS Appl. Mater. Interfaces, 2018, 10, 193-206.

42 L. Mei, X. Gao, Y. Shi, C. Cheng, Z. Shi, M. Jiao, F. Cao, $\mathrm{Z}$. $\mathrm{Xu}, \mathrm{X}$. Li and J. Zhang, Augmented graphene quantum dot-light irradiation therapy for bacteria-infected wounds, ACS Appl. Mater. Interfaces, 2020, 12, 40153-40162.

43 Z. Wang, T. Hu and R. Liang, and Min Wei, Application of zero-dimensional nanomaterials in biosensing, Front. Chem., 2020, 8, 320-339.

44 C. Wang, H. Hong, Z. Lin, Y. Yuan, C. Liu, X. Ma and $\mathrm{X}$. Cao, Tethering silver ions on amino-functionalized mesoporous silica for enhanced and sustained antibacterial properties, $R S C$ Adv., 2015, 5, 104289-104298.

45 S. Pal, E. J. Yoon, Y. K. Tak, E. C. Choi and J. M. Song, Synthesis of highly antibacterial nanocrystalline trivalent 
silver polydiguanide, J. Am. Chem. Soc., 2009, 131, 16147-16155.

46 A. Burdusel, O. Gherasim, A. M. Grumezescu, L. Mogoantă, A. Ficai and E. Andronescu, Biomedical applications of silver nanoparticles: an up-to-date overview, Nanomaterials, 2018, 8, 681-706.

47 G. Franci, A. Falanga, S. Galdiero, L. Palomba, M. Rai, G. Morelli and M. Galdiero, Silver nanoparticles as potential antibacterial agents, Molecules, 2015, 20, 8856-8874.

48 S. Wang, J. Sun, Y. Jia, L. Yang, N. Wang, Y. Xianyu, W. Chen, X. Li, R. Cha and X. Jiang, Nanocrystalline cellulose-assisted generation of silver nanoparticles for nonenzymatic glucose detection and antibacterial agent, Biomacromolecules, 2016, 17, 2472-2478.

49 N. M. Huang, S. Radiman, H. N. Lim, P. S. Khiew, W. S. Chiu, K. H. Lee, A. Syahida, R. Hashim and C. H. Chia, $\gamma$-Ray assisted synthesis of silver nanoparticles in chitosan solution and the antibacterial properties, Chem. Eng. J., 2009, 15, 499-507.

50 L. Huang, Y. Lou, D. Zhang, L. Ma, H. Qian, Y. Hu, P. Ju, D. $\mathrm{Xu}$ and $\mathrm{X}$. Li, D-Cysteine functionalised silver nanoparticles surface with a "disperse-then-kill" antibacterial synergy, Chem. Eng. J., 2020, 381, 122662.

51 C. Cao, W. Ge, J. Yin, D. Yang, W. Wang, X. Song, Y. Hu, J. Yin and X. Dong, Mesoporous silica supported silverbismuth nanoparticles as photothermal agents for skin infection synergistic antibacterial therapy, Small, 2020, 16, 2000436.

52 R. Prucek, J. Tuček, M. Kilianová, A. Panáček, L. Kvítek, J. Filip, M. Kolář, K. Tománková and R. Zbořil, The targeted antibacterial and antifungal properties of magnetic nanocomposite of iron oxide and silver nanoparticles, Biomaterials, 2011, 32, 4704-4713.

53 L. Zhao, H. Wang, K. Huo, L. Cui, W. Zhang, H. Ni, Y. Zhang, Z. Wu and P. K. Chu, Antibacterial nanostructured titania coating incorporated with silver nanoparticles, Biomaterials, 2011, 32, 5706-5716.

54 S. Shakya, Y. He, X. Ren, T. Guo, A. Maharjan, T. Luo, T. Wang, R. Dhakhwa, B. Regmi, H. Li, R. Gref and J. Zhang, Ultrafine silver nanoparticles embedded in cyclodextrin metal-organic frameworks with GRGDS functionalization to promote antibacterial and wound healing application, Small, 2019, 15, 1901065.

55 S. Tavakoli and A. S. Klar, Advanced hydrogels as wound dressings, Biomolecules, 2020, 10, 1169-1189.

56 S. Li, S. Dong, W. Xu, S. Tu, L. Yan, C. Zhao, J. Ding and X. Chen, Antibacterial hydrogels, Adv. Sci., 2018, 5, 1700527.

57 T. Dai, C. Wang, Y. Wang, W. Xu, J. Hu and Y. Cheng, A nanocomposite hydrogel with potent and broad-spectrum antibacterial activity, ACS Appl. Mater. Interfaces, 2018, 10, 15163-15173.

58 H. Fang, J. Wang, L. Li, L. Xu, Y. Wu, Y. Wang, X. Fei, J. Tian and Y. Li, A novel high-strength poly (ionic liquid)/ PVA hydrogel dressing for antibacterial applications, Chem. Eng. J., 2019, 365, 153-164.
59 B. L. Patenall, G. T. Williams, L. Gwynne, L. J. Stephens, E. V. Lampard, H. J. Hathaway, N. T. Thet, A. E. Young, M. J. Sutton, R. D. Short, S. D. Bull, T. D. James, A. C. Sedgwick and T. A. Jenkins, Reaction-based indicator displacement assay (RIA) for the development of a triggered release system capable of biofilm inhibition, Chem. Commun., 2019, 55, 15129-15132.

60 Z. Chen, M. Mo, F. Fu, L. Shang, H. Wang, C. Liu and Y. Zhao, Antibacterial structural color hydrogels, ACS Appl. Mater. Interfaces, 2017, 9, 38901-38907.

61 E. E. Connor, J. Mwamuka, A. Gole, C. J. Murphy and M. D. Wyatt, Gold nanoparticles are taken up by human cells but do not cause acute cytotoxicity, Small, 2005, 1, 325-327.

62 B. Purohit, A. Kumar, K. Mahato and P. Chandra, Novel sensing assembly comprising engineered gold dendrites and MWCNT-AuNPs nanohybrid for acetaminophen detection in human urine, Electroanalysis, 2020, 32, 561-570.

63 X. An, F. Zhan and Y. Zhu, Smart photothermal-triggered bilayer phase transition in AuNPs-liposomes to release drug, Langmuir, 2013, 29, 1061-1068.

64 Y. Zhao, Y. Tian, Y. Cui, W. Liu, W. Ma and X. Jiang, Small molecule-capped gold nanoparticles as potent antibacterial agents that target Gram-negative bacteria, J. Am. Chem. Soc., 2010, 132, 12349-12356.

65 A. Rai, A. Prabhune and C. C. Perry, Antibiotic mediated synthesis of gold nanoparticles with potent antimicrobial activity and their application in antimicrobial coatings, J. Mater. Chem., 2010, 20, 6789-6798.

66 C. Zhang, D.-T. Shi, K.-C. Yan, A. C. Sedgwick, G.-R. Chen, X.-P. He, T. D. James, B. Ye, X.-L. Hu and D. Chen, A glycoconjugate-based gold nanoparticle approach for the targeted treatment of Pseudomonas aeruginosa biofilms, Nanoscale, 2020, 12, 23234-23240.

67 Y. Xie, J. Yang, J. Zhang, W. Zheng and X. Jiang, Activating antibacterial effect of 4,6-diamino-2-pyrimidinethiolmodified gold nanoparticles by reducing their sizes, Angew. Chem., Int. Ed., 2020, 59, 23471-23475.

68 Y. Feng, W. Chen, Y. Jia, Y. Tian, Y. Zhao, F. Long, Y. Rui and $\mathrm{X}$. Jiang, N-Heterocyclic molecule-capped gold nanoparticles as effective antibiotics against multi-drug resistant bacteria, Nanoscale, 2016, 8, 13223-13227.

69 Y. Li, Y. Tian, W. Zheng, Y. Feng, R. Huang, J. Shao, R. Tang, P. Wang, Y. Jia, J. Zhang, W. Zheng, G. Yang and $\mathrm{X}$. Jiang, Composites of bacterial cellulose and small molecule-decorated gold nanoparticles for treating Gramnegative bacteria-infected wounds, Small, 2017, 13, 1700130.

70 H. Qiu, F. Pu, Z. Liu, Q. Deng, P. Sun, J. Ren and X. Qu, Depriving bacterial adhesion-related molecule to inhibit biofilm formation using $\mathrm{CeO}_{2}$-decorated metal-organic frameworks, Small, 2019, 15, 1902522.

71 J. Liu, S. Lu, Q. Tang, K. Zhang, W. Yu, H. Sun and B. Yang, One-step hydrothermal synthesis of photoluminescent carbon nanodots with selective antibacterial activity 
against Porphyromonas gingivalis, Nanoscale, 2017, 9, 7135-7142.

72 E. Hoshino, N. Kurihara-Ando, I. Sato, H. Uematsu, M. Sato, K. Kota and M. Iwaku, In vitro antibacterial susceptibility of bacteria taken from infected root dentine to a mixture of ciprofloxacin, metronidazole and minocycline, Int. Endodont. J., 1996, 29, 125-130.

73 W. Bing, H. Sun, Z. Yan, J. Ren and X. Qu, Programmed bacteria death induced by carbon dots with different surface charge, Small, 2016, 12, 4713-4718.

74 L. Hui, J. Huang, G. Chen, Y. Zhu and L. Yang, Antibacterial property of graphene quantum dots (both source material and bacterial shape matter), ACS Appl. Mater. Interfaces, 2016, 8, 20-25.

75 H. Han, J. Zhu, D.-Q. Wu, F.-X. Li, X.-L. Wang, J.-Y. Yu and $\mathrm{X}$.-H. Qin, Inherent guanidine nanogels with durable antibacterial and bacterially antiadhesive properties, $A d v$. Funct. Mater., 2019, 29, 1806594.

76 X. Yan, Z. Jie, L. Zhao, H. Yang, S. Yang and J. Liang, Highefficacy antibacterial polymeric micro/nano particles with $\mathrm{N}$ halamine functional groups, Chem. Eng. J., 2014, 254, 30-38.

77 R. Zhao, H. Wang, T. Ji, G. Anderson, G. Nie and Y. Zhao, Biodegradable cationic e-poly-L-lysine-conjugated polymeric nanoparticles as a new effective antibacterial agent, Sci. Bull., 2015, 60, 216-226.

78 H. J. Zhang, Y. C. Liang, H. Zhao, R. L. Qi, Z. Chen, H. X. Yuan, H. Y. Liang and L. Wang, Dual-mode antibacterial conjugated polymer nanoparticles for photothermal and photodynamic therapy, Macromol. Biosci., 2019, 20, 1900301.

79 L. X. Guo, H. P. Wang, Y. X. Wang, F. Liu and L. H. Feng, Organic polymer nanoparticles with primary ammonium salt as potent antibacterial nanomaterials, ACS Appl. Mater. Interfaces, 2020, 12, 21254-21262.

80 D. K. Li, Y. S. Fang and X. M. Zhang, Bacterial detection and elimination using a dual-functional porphyrin-based porous organic polymer with peroxidase-like and high near-infrared-light-enhanced antibacterial activity, ACS Appl. Mater. Interfaces, 2020, 12, 8989-8999.

81 Z. W. Zhang, M. M. Jones, C. Sabatini, S. T. Vanyo, M. Yang, A. Kumar, Y. C. Jiang, M. T. Swihart, M. B. Visser and C. Cheng, Synthesis and antibacterial activity of polymer-antibiotic conjugates incorporated into a resin-based dental adhesive, Biomater. Sci., 2021, 9, 2043-2052.

82 Y. Xia, P. Yang, Y. Sun, Y. Wu, B. Mayers, B. Gates, Y. Yin, F. Kim and H. Yan, One-dimensional nanostructures: Synthesis, characterization, and applications, Adv. Mater., 2003, 15, 353-389.

83 L. Zhang, S. Sirivisoot, G. Balasundaram and T. J. Webster, Nanomaterials for improved orthopedic and bone tissue engineering applications, Adv. Biomater., 2009, 205-241.

84 X.-L. Hu, N. Kwon, K.-C. Yan, A. C. Sedgwick, G.-R. Chen, X.-P. He, T. D. James and J. Yoon, Bio-conjugated advanced materials for targeted disease theranostics, Adv. Funct. Mater., 2020, 30, 1907906.
85 G. V. Vimbela, S. M. Ngo, C. Fraze, L. Yang and D. A. Stout, Antibacterial properties and toxicity from metallic nanomaterials, Int. J. Nanomed., 2017, 12, 3941-3965.

86 J.-H. Kim, J. Ma, S. Jo, S. Lee and C. S. Kim, Enhancement of antibacterial properties of a silver nanowire film via electron beam irradiation, ACS Appl. Bio Mater., 2020, 3, 2117-2124.

87 L. Qin, P. Wang, Y. Guo, C. Chen and M. Liu, Selfassembled soft nanomaterials via silver(I)-coordination: Nanotube, nanofiber, and remarkably enhanced antibacterial effect, Adv. Sci., 2015, 2, 1500134.

88 F. Gao, H. Pang, S. Xu and Q. Lu, Copper-based nanostructures: Promising antibacterial agents and photocatalysts, Chem. Commun., 2009, 3571-3573.

89 A. Rauf, J. Ye, S. Zhang, Y. Qi, G. Wang, Y. Che and G. Ning, Copper(II)-based coordination polymer nanofibers as a highly effective antibacterial material with a synergistic mechanism, Dalton Trans., 2019, 48, 17810-17817.

90 Z. Zhang, J. Wang and C. Chen, Gold nanorods based platforms for light-mediated theranostics, Theranostics, 2013, 3, 223-238.

91 Y.-Q. Zhao, Y. Sun, Y. Zhang, X. Ding, N. Zhao, B. Yu, H. Zhao, S. Duan and F.-J. Xu, Well-defined gold nanorod/ polymer hybrid coating with inherent antifouling and photothermal bactericidal properties for treating an infected hernia, ACS Nano, 2020, 14, 2265-2275.

92 W.-N. Wang, P. Pei, Z.-Y. Chu, B.-J. Chen, H.-S. Qian, Z.-B. Zha, W. Zhou, T. Liu, M. Shao and H. Wang, Bi2S3 coated $\mathrm{Au}$ nanorods for enhanced photodynamic and photothermal antibacterial activities under NIR light, Chem. Eng. J., 2020, 397, 125488.

93 Q. Chen, C. Wei, F. Gao, H. Pang and Q. Lu, Bi-directionalbi-dimensionality alignment of self-supporting $\mathrm{Mn3O} 4$ nanorod and nanotube arrays with different bacteriostasis and magnetism, Nanoscale, 2013, 5, 12231-12236.

94 M. S. Selim, H. Hamouda, Z. Hao, S. Shabana and X. Chen, Design of $\gamma-\mathrm{AlOOH}, \gamma-\mathrm{MnOOH}$, and $\alpha-\mathrm{Mn} 2 \mathrm{O} 3$ nanorods as advanced antibacterial active agents, Dalton Trans., 2020, 49, 8601-8613.

95 N. Habibi, N. Kamaly, A. Memic and H. Shafiee, Selfassembled peptide-based nanostructures: Smart nanomaterials toward targeted drug delivery, Nano Today, 2016, 11, 41-60.

96 Y. Wang, C. Chen, D. Zhang and J. Wang, Bifunctionalized novel Co-V MMO nanowires: Intrinsic oxidase and peroxidase like catalytic activities for antibacterial application, Appl. Catal., B, 2020, 261, 118256.

97 D. W. Horn, G. Ao, M. Maugey, C. Zakri, P. Poulin and V. A. Davis, Dispersion state and fiber toughness: Antibacterial lysozyme-single walled carbon nanotubes, $A d v$. Funct. Mater., 2013, 23, 6082-6090.

98 L. Lei, D. Huang, G. Zeng, M. Cheng, D. Jiang, C. Zhou, S. Chen and W. Wang, A fantastic two-dimensional MoS2 material based on the inert basal planes activation: Electronic structure, synthesis strategies, catalytic active sites, catalytic and electronics properties, Coord. Chem. Rev., 2019, 399, 213020. 
99 D. P. Linklater, V. A. Baulin, S. Juodkazis and E. P. Ivanova, Mechano-bactericidal mechanism of graphene nanomaterials, Interface Focus, 2018, 8, 20170060.

100 V. Georgakilas, J. N. Tiwari, K. C. Kemp, J. A. Perman, A. B. Bourlinos, K. S. Kim and R. Zboril, Noncovalent functionalization of graphene and graphene oxide for energy materials, biosensing, catalytic, and biomedical applications, Chem. Rev., 2016, 11, 5464-5519.

101 X. P. Hao, S. G. Chen, W. H. Wang, Z. Q. Yang, L. F. Yue, H. Y. Sun and F. Cheng, AgNP-Coordinated glucosaminegrafted carbon nanotubes with enhanced antibacterial properties, New J. Chem., 2017, 41, 7045-7051.

102 Y. Huang, W.-T. Dou, F. Xu, H.-B. Ru, Q. Gong, D. Wu, D. Yan, H. Tian, X.-P. He, Y. Mai and X. Feng, Supramolecular nanostructures of structurally defined graphene nanoribbons in the aqueous phase, Angew. Chem., Int. Ed., 2018, 57, 3366-3371.

103 Z.-H. Yu, X. S. Li, F. G. Xu, X.-L. Hu, J. T. Yan, N. Kwon, G.-R. Chen, T. T. Tang, X. J. Dong, Y. Y. Mai, D. J. Chen, J. Yoon, X.-P. He and H. Tian, A supramolecular-based dual-wavelength phototherapeutic agent with broadspectrum antimicrobial activity against drug-resistant bacteria, Angew. Chem., Int. Ed., 2020, 59, 3658-3664.

104 C. Tan, X. Cao, X.-J. Wu, Q. He, J. Yang, X. Zhang, J. Chen, W. Zhao, S. Han, G.-H. Nam, M. Sindoro and H. Zhang, Recent advances in ultrathin two-dimensional nanomaterials, Chem. Rev., 2017, 117, 6225-6331.

105 H. Moon, J. Bang, S. Hong, G. Kim, J. W. Roh, J. Kim and W. Lee, Strong thermopower enhancement and tunable power factor via semimetal to semiconductor transition in a transition-metal dichalcogenide, ACS Nano, 2019, 13, 13317-13324.

106 L. Mei, S. Zhu, W. Yin, C. Chen, G. Nie, Z. Gu and Y. Zhao, Two-dimensional nanomaterials beyond graphene for antibacterial applications: Current progress and future perspectives, Theranostics, 2020, 10, 757-781.

107 X. Y. Wong, A. Sena-Torralba, R. Álvarez-Diduk, K. Muthoosamy and A. Merkoci, Nanomaterials for nanotheranostics: Tuning their properties according to disease needs, ACS Nano, 2020, 14, 2585-2627.

108 S. Sajjad, S. A. K. Leghari and A. Iqbal, Study of graphene oxide structural features for catalytic, antibacterial, gas sensing, and metals decontamination environmental applications, ACS Appl. Mater. Interfaces, 2017, 9, 43393-43414.

109 I. Sengupta, P. Bhattacharya, M. Talukdar, S. Neogi, S. K. Pal and S. Chakraborty, Bactericidal effect of graphene oxide and reduced graphene oxide: Influence of shape of bacteria, Colloid Interface Sci. Commun., 2019, 28, 60-68.

110 M. T. H. Aunkor, T. Raihan, S. H. Prodhan, H. S. C. Metselaar, S. U. F. Malik and A. K. Azad, Antibacterial activity of graphene oxide nanosheet against multidrug resistant superbugs isolated from infected patients, R. Soc. Open Sci., 2020, 7, 200640.

111 J. He, X. Zhu, Z. Qi, L. Wang, A. Aldalbahi, J. Shi, S. Song, C. Fan, M. Lv and Z. Tang, The inhibition effect of graphene oxide nanosheets on the development of streptococcus mutans biofilms, Part. Part. Syst. Charact., 2017, 34, 1700001.

112 L. Zhang, P. Chen, Y. Xu, W. Nie and Y. Zhou, Enhanced photo-induced antibacterial application of graphene oxide modified by sodium anthraquinone-2-sulfonate under visible light, Appl. Catal., B, 2020, 265, 118572.

113 Y. Ohta, Y. Kondo, K. Kawada, T. Teranaka and N. Yoshino, Synthesis and antibacterial activity of quaternary ammonium salt-type antibacterial agents with a phosphate group, J. Oleo Sci., 2008, 57, 445-452.

114 S. K. Tiwari, X. Guo, Y. Huang, X. Zhou, H. H. K. Xu, B. Ren, X. Peng, M. D. Weir, M. Li and L. Cheng, The inhibitory effect of quaternary ammonium salt on bacteria in root canal, Sci. Rep., 2019, 9, 12463-12473.

115 X. Ye, X. Qin, X. Yan, J. Guo, L. Huang, D. Chen, T. Wu, Q. Shi, S. Tan and X. Cai, $\pi-\pi$ conjugations improve the long-term antibacterial properties of graphene oxide/quaternary ammonium salt nanocomposites, Chem. Eng. J., 2016, 304, 873-881.

116 X. Cai, S. Tan, A. Yu, J. Zhang, J. Liu, W. Mai and Z. Jiang, Sodium 1-naphthalenesulfonate-functionalized reduced graphene oxide stabilizes silver nanoparticles with lower cytotoxicity and long-term antibacterial activity, Chem. Asian J., 2012, 7, 1664-1670.

117 R. Zhao, M. Lv, Y. Li, M. Sun, W. Kong, L. Wang, S. Song, C. Fan, L. Jia, S. Qiu, Y. Sun, H. Song and R. Hao, Stable nanocomposite based on PEGylated and silver nanoparticles loaded graphene oxide for long-term antibacterial activity, ACS Appl. Mater. Interfaces, 2017, 9, 15328-15341.

118 S. Ma, S. Zhan, Y. Jia and Q. Zhou, Highly efficient antibacterial and $\mathrm{Pb}(\mathrm{II})$ removal effects of $\mathrm{Ag}-\mathrm{CoFe}_{2} \mathrm{O}_{4}-$ GO nanocomposite, ACS Appl. Mater. Interfaces, 2015, 7, 10576-10586.

119 T. I. Kim, B. Kwon, J. Yoon, I.-J. Park, G. S. Bang, Y. Park, Y. Seo and S. Choi, Antibacterial activities of graphene oxide-molybdenum disulfide nanocomposite films, ACS Appl. Mater. Interfaces, 2017, 9, 7908-7917.

120 H. Cui, Z. Gu, X. Chen, L. Lin, Z. Wang, X. Dai, Z. Yang, L. Liu, R. Zhou and M. Dong, Stimulating antibacterial activities of graphitic carbon nitride nanosheets with plasma treatment, Nanoscale, 2019, 11, 18416-18425.

121 V. Yadav, S. Roy, P. Singh, Z. Khan and A. Jaiswal, 2D MoS2-based nanomaterials for therapeutic, bioimaging, and biosensing applications, Small, 2019, 15, 1803706.

122 J. Niu, Y. Sun, F. Wang, C. Zhao, J. Ren and X. Qu, Photomodulated nanozyme used for a Gram-selective antimicrobial, Chem. Mater., 2018, 30, 7027-7033.

123 Y. Huang, Q. Gao, X. Li, Y. Gao, H. Han, Q. Jin, K. Yao and J. Ji, Ofloxacin loaded MoS2 nanoflakes for synergistic mild-temperature photothermal/antibiotic therapy with reduced drug resistance of bacteria, Nano Res., 2020, 13, 2340-2350.

124 P. Pacher, J. S. Beckman and L. Liaudet, Nitric oxide and peroxynitrite in health and disease, Physiol. Rev., 2007, 87, 315-424. 
125 M. Weber, H.-H. Han, B.-H. Li, M. L. Odyniec, C. E. F. Jarman, Y. Zang, S. D. Bull, A. B. Mackenzie, A. C. Sedgwick, J. Li, X.-P. He and T. D. James, Pinkment: A synthetic platform for the development of fluorescent probes for diagnostic and theranostic applications, Chem. Sci., 2020, 11, 8567-8571.

126 Q. Gao, X. Zhang, W. Yin, D. Ma, C. Xie, L. Zheng, X. Dong, L. Mei, J. Yu, C. Wang, Z. Gu and Y. Zhao, Functionalized MoS2 nanovehicle with near-infrared laser-mediated nitric oxide release and photothermal activities for advanced bacteria-infected wound therapy, Small, 2018, 14, 1802290.

127 F. Cao, E. Ju, Y. Zhang, Z. Wang, C. Liu, W. Li, Y. Huang, K. Dong, J. Ren and X. Qu, An efficient and benign antimicrobial depot based on silver-infused $\mathrm{MoS}_{2}$, ACS Nano, 2017, 11, 4651-4659.

128 W. Zhu, X. Liu, L. Tan, Z. Cui, X. Yang, Y. Liang, Z. Li, S. Zhu, K. W. K. Yeung and S. Wu, AgBr nanoparticles in situ growth on 2D $\mathrm{MoS}_{2}$ nanosheets for rapid bacteriakilling and photodisinfection, ACS Appl. Mater. Interfaces, 2019, 11, 34364-34375.

129 H. Lin, Y. Chen and J. Shi, Insights into 2D MXenes for versatile biomedical applications: Current advances and challenges ahead, Adv. Sci., 2018, 5, 1800518.

130 K. Rasool, M. Helal, A. Ali, C. E. Ren, Y. Gogotsi and K. A. Mahmoud, Antibacterial activity of $\mathrm{Ti}_{3} \mathrm{C}_{2} \mathrm{~T}_{x}$ MXene, ACS Nano, 2016, 10, 3674-3684.

131 Z. Miao, L. Fan, X. Xie, Y. Ma, J. Xue, T. He and Z. Zha, Liquid exfoliation of atomically thin antimony selenide as an efficient two-dimensional antibacterial nanoagent, ACS Appl. Mater. Interfaces, 2019, 11, 26664-26673.

132 P. Cheng, Y. Chen, X. Yan, Y. Wang and W.-Z. Lang, Highly stable and antibacterial two-dimensional tungsten disulfide lamellar membrane for water filtration, ChemSusChem, 2019, 12, 275-282.

133 A. Pramanik, D. Davis, S. Patibandla, S. Begum, P. Ray, K. Gates, Y. Gao and P. C. Ray, A WS $\mathrm{W}_{2}$-gold nanoparticle heterostructure-based novel SERS platform for the rapid identification of antibiotic-resistant pathogens, Nanoscale Adv., 2020, 2, 2025-2033.

134 M. Ikram, R. Tabassum, U. Qumar, S. Ali, A. Ul-Hamid, A. Haider, A. Haider, A. Raza, M. Imran and S. Ali, Promising performance of chemically exfoliated Zr-doped $\mathrm{MoS}_{2}$ nanosheets for catalytic and antibacterial applications, RSC Adv., 2020, 10, 20559-20571.

135 D. Ma, C. Xie, T. Wang, L. Mei, X. Zhang, Z. Guo and W. Yin, Liquid-phase exfoliation and functionalization of $\mathrm{MoS}_{2}$ nanosheets for effective antibacterial application, ChemBioChem, 2020, 21, 2373-2380.

136 Z. Feng, X. Liu, L. Tan, Z. Cui, X. Yang, Z. Li, Y. Zheng, K. W. K. Yeung and S. Wu, Electrophoretic deposited stable chitosan@ $\mathrm{MoS}_{2}$ coating with rapid in situ bacteria-killing ability under dual-light irradiation, Small, 2018, 14, 1704347.

137 X. Zhao, M. Chen, H. Wang, L. Xia, M. Guo, S. Jiang, Q. Wang, X. Li and X. Yang, Synergistic antibacterial activity of streptomycin sulfate loaded PEG-MoS $2 /$ rGO nanoflakes assisted with near-infrared, Mater. Sci. Eng., C, 2020, 116, 111221.

138 İ. Aksoy, H. Küçükkeçeci, F. Sevgi, Ö. Metin and I. H. Patir, Photothermal antibacterial and antibiofilm activity of black phosphorus/gold nanocomposites against pathogenic bacteria, ACS Appl. Mater. Interfaces, 2020, 12, 26822-26831.

139 J. N. Tiwari, R. N. Tiwari and K. S. Kim, Zero-dimensional, one-dimensional, two-dimensional and three-dimensional nanostructured materials for advanced electrochemical energy devices, Prog. Mater. Sci., 2012, 57, 724-803.

140 D. Li, G. Wang, L. Cheng, C. Wang and X. Mei, Engineering the self-assembly induced emission of copper nanoclusters as $3 \mathrm{D}$ nanomaterials with mesoporous sphere structures by the crosslinking of Ce3+, ACS Omega, 2018, 3, 14755-14765.

141 M. Shen, F. Forghani, X. Kong, D. Liu, X. Ye, S. Chen and T. Ding, Antibacterial applications of metal-organic frameworks and their composites, Compr. Rev. Food Sci. Food Saf., 2020, 19, 1397-1419.

142 J. Shi, X. Du, Y. Huang, J. Zhou, D. Yuan, D. Wu, Y. Zhang, R. Haburcak, I. R. Epstein and B. Xu, Ligand-receptor interaction catalyzes the aggregation of small molecules to induce cell necroptosis, J. Am. Chem. Soc., 2015, 137, 26-29.

143 L. Zhang, Z. Liu, Q. Deng, Y. J. Sang, K. Dong, J. Ren and $\mathrm{X}$. Qu, Nature-inspired construction of MOF@COF nanozyme with active sites in tailored microenvironment and pseudopodia-like surface for enhanced bacterial inhibition, Angew. Chem., Int. Ed., 2021, 133, 3511-3516.

144 J.-C. Kung, W.-H. Wang, C.-L. Lee, H.-C. Hsieh and C.-J. Shih, Antibacterial activity of silver nanoparticles (AgNP) confined to mesostructured, silica-based calcium phosphate against methicillin-resistant staphylococcus aureus (MRSA), Nanomaterials, 2020, 10, 1264-1277.

145 C. Shuai, Y. Xu, P. Feng, G. Wang, S. Xiong and S. Peng, Antibacterial polymer scaffold based on mesoporous bioactive glass loaded with in situ grown silver, Chem. Eng. J., 2019, 374, 304-315.

146 K. Xiong, J. Li, L. Tan, Z. D. Cui, Z. Y. Li, S. L. Wu, Y. Q. Liang, S. G. Zhu and X. M. Liu, Ag2S decorated nanocubes with enhanced near-infrared photothermal and photodynamic properties for rapid sterilization, Colloid Interface Sci. Commun., 2019, 33, 100201.

147 X. Y. Zhang, G. N. Zhang, M. Z. Chai, X. H. Yao, W. Y. Chen and P. K. Chu, Synergistic antibacterial activity of physicalchemical multi-mechanism by $\mathrm{TiO}_{2}$ nanorod arrays for safe biofilm eradication on implant, Bioact. Mater., 2021, 6, 12-25.

148 Y. Song, L. Cai, Z. Tian, Y. Wu and J. Chen, Phytochemical curcumin-coformulated, silver-decorated melanin-like polydopamine/mesoporous silica composites with improved antibacterial and chemotherapeutic effects against drugresistant cancer cells, ACS Omega, 2020, 5, 15083-15094.

149 D. Xia, X. Wang, Y. Wang, Y. Wang, H. Meng, L. Li, P. Zhou and $\mathrm{S}$. Xu, Silver-decorated mesostructured cellular silica 
foams as excellent antibacterial hemostatic agents for rapid and effective treatment of hemorrhage, Mater. Sci. Eng., C, 2020, 115, 111105.

150 X. Wang, R. Li, Z. Li, R. Xiao, X.-B. Chen and T. Zhang, Design and preparation of nanoporous $\mathrm{Ag}-\mathrm{Cu}$ alloys by dealloying $\mathrm{Mg}-(\mathrm{Ag}, \mathrm{Cu})-\mathrm{Y}$ metallic glasses for antibacterial applications, J. Mater. Chem. B, 2019, 7, 4169-4176.

151 J. Luo, J. Lai, N. Zhang, Y. Liu, R. Liu and X. Liu, Tannic acid induced self-assembly of three-dimensional graphene with good adsorption and antibacterial properties, ACS Sustainable Chem. Eng., 2016, 4, 1404-1413.

152 R. Liu, H. Ge, X. Wang, J. Luo, Z. Li and X. Liu, Threedimensional Ag-tannic acid-graphene as an antibacterial material, New J. Chem., 2016, 40, 6332-6339.

153 E. P. Komarala and S. Doshi, A. Mohammed and D. Bahadur, Efficient antibacterial activity via protein degradation of a 3D layered double hydroxide-reduced graphene oxide nanohybrid, RSC Adv., 2016, 6, 40389-40398.

154 L. W. Wang, F. Gao, A. Z. Wang, X. Y. Chen, H. Li, X. Zhang, H. Zheng, R. Ji, B. Li, X. Yu, J. Liu, Z. J. Gu, F. L. Chen and C. Y. Chen, Defect-rich adhesive molybdenum disulfide/rGO vertical heterostructures with enhanced nanozyme activity for smart bacterial killing application, Adv. Mater., 2020, 32, 2005423.

155 X. Li, Y. Wang, M. Guo, Z. Wang, N. Shao, P. Zhang, $\mathrm{X}$. Chen and Y. Huang, Degradable three dimensionalprinted polylactic acid scaffold with long-term antibacterial activity, ACS Sustain, Chem. Eng., 2018, 6, 2047-2054.

156 N. Yan, Y. Zhou, Y. Zheng, S. Qiao, Q. Yu, Z. Li and H. Lu, Antibacterial properties and cytocompatibility of bio-based nanostructured carbon aerogels derived from silver nanoparticles deposited onto bacterial cellulose, RSC Adv., 2015, 5, 97467-97476.

157 S. Zhou, Z. Wang, Y. Wang and L. Feng, Near-infrared light-triggered synergistic phototherapy for antimicrobial therapy, ACS Appl. Bio Mater., 2020, 3, 1730-1737.
158 S. Rigo, C. Cai, G. Gunkel-Grabole, L. Maurizi, X. Y. Zhang, J. $\mathrm{Xu}$ and C. G. Palivan, Nanoscience-based strategies to engineer antimicrobial surfaces, Adv. Sci., 2018, 5, 1700892.

159 T. Wei, Q. Yu and H. Chen, Responsive and synergistic antibacterial coatings: Fighting against bacteria in a smart and effective way, Adv. Healthcare Mater., 2019, 8, 1801381.

160 W. B. Hu, C. Peng, W. J. Luo, M. Lv, X. M. Li, D. Li, Q. Huang and C. H. Fan, Graphene-Based Antibacterial Paper, ACS Nano, 2010, 4, 4317-4323.

161 J. M. Zhao, B. Deng, M. Lv, J. Y. Li, Y. J. Zhang, H. Q. Jiang, C. Peng, J. Li, J. Y. Shi, Q. Huang and C. H. Fan, Graphene oxide-based antibacterial cotton fabrics, Adv. Healthcare Mater., 2013, 2, 1259-1266.

162 R. Zhang, Y. Kang and B. Xie, Assembly and antibacterial activity of horizontally oriented silver nanoplates, J. Appl. Polym. Sci., 2015, 132, 42070.

163 L. A. Rank, N. M. Walsh, R. Liu, F. Y. Lim, J. W. Bok, M. Huang, N. P. Keller, S. H. Gellman and C. M. Hull, A cationic polymer that shows high antifungal activity against diverse human pathogens, Antimicrob. Agents Chemother., 2017, 61, 204-217.

164 H. Choi, S. Chakraborty, R. Liu, S. H. Gellman and J. C. Weisshaar, Single-cell, time-resolved antimicrobial effects of a highly cationic, random Nylon-3 copolymer on live escherichia coli, ACS Chem. Biol., 2016, 11, 113-120.

165 H. Etayash, Y. Qian, D. Pletzer, Q. Zhang, J. Xie, R. Cui, C. Dai, P. Ma, F. Qi, R. Liu and R. E. W. Hancock, Host defense peptide-mimicking amphiphilic $\beta$-peptide polymer (Bu:DM) exhibiting anti-biofilm, immunomodulatory, and in vivo anti-infective activity, J. Med. Chem., 2020, 63, 12921-12928.

166 W. T. Dou, Z. Y. Qin, J. Li, D. M. Zhou and X. P. He, Sci. Bull., 2019, 64(24), 1902-1909. 